\title{
THE MID-INFRARED HIGH-IONIZATION LINES FROM ACTIVE GALACTIC NUCLEI AND STAR-FORMING GALAXIES*
}

\author{
Miguel Pereira-Santaella ${ }^{1,2}$, Aleksandar M. Diamond-Stanic $^{3}$, Almudena Alonso-Herrero $^{1,2,4}$, \\ AND GEORGE H. RIEKE ${ }^{3}$ \\ ${ }^{1}$ Instituto de Estructura de la Materia, CSIC, Serrano 121, E-28006 Madrid, Spain; pereira@damir.iem.csic.es \\ ${ }^{2}$ Departamento de Astrofísica, Centro de Astrobiología, CSIC/INTA, Carretera de Torrejón a Ajalvir, km 4, 28850 Torrejón de Ardoz, Madrid, Spain \\ ${ }^{3}$ Steward Observatory, University of Arizona, 933 North Cherry Avenue, Tucson, AZ 85721, USA \\ Received 2010 May 5; accepted 2010 October 10; published 2010 December 6
}

\begin{abstract}
We used Spitzer/Infrared Spectrograph spectroscopic data on 426 galaxies including quasars, Seyferts, LINERs, and $\mathrm{H}$ II galaxies to investigate the relationship among the mid-IR emission lines. There is a tight linear correlation between the $[\mathrm{Ne} \mathrm{v}] 14.3 \mu \mathrm{m}$ and $24.3 \mu \mathrm{m}(97.1 \mathrm{eV})$ and the $[\mathrm{O}$ IV] $25.9 \mu \mathrm{m}(54.9 \mathrm{eV})$ high-ionization emission lines. The correlation also holds for these high-ionization emission lines and the [Ne III] $15.56 \mu \mathrm{m}(41 \mathrm{eV})$ emission line, although only for active galaxies. We used these correlations to calculate the [Ne III] excess due to star formation in Seyfert galaxies. We also estimated the [O IV] luminosity due to star formation in active galaxies and determined that it dominates the [O IV] emission only if the contribution of the active nucleus to the total luminosity is below 5\%. We find that the active galactic nucleus dominates the [O IV] emission in most Seyfert galaxies, whereas star formation adequately explains the observed [O IV] emission in optically classified H II galaxies. Finally, we computed photoionization models to determine the physical conditions of the narrow-line region where these high-ionization lines originate. The estimated ionization parameter range is $-2.8<\log U<-2.5$ and the total hydrogen column density range is $20<\log n_{\mathrm{H}}\left(\mathrm{cm}^{-2}\right)<21$.
\end{abstract}

Key words: galaxies: active - galaxies: nuclei - galaxies: starburst - infrared: galaxies

Online-only material: color figures, machine-readable table

\section{INTRODUCTION}

Primarily through measurements in the optical, active galactic nuclei (AGNs) have been categorized as type 1 or 2, respectively, with and without very broad emission lines. The unification model (Antonucci 1993; Urry \& Padovani 1995) successfully explains this behavior in terms of a dusty circumnuclear torus that hides the broad-line region (BLR) from our line of sight for type 2 AGNs. It also appears that extinction in the host galaxy can hide the BLR (e.g., Maiolino \& Rieke 1995; AlonsoHerrero et al. 2003) in some active galaxies. Extinction therefore affects the optical properties of AGN in fundamental ways. These effects can be minimized by studying these objects using infrared (IR) emission lines, both to test the predictions of the unification model and to characterize AGNs in a uniform way. Toward this end, we have used mid-IR observations with the Infrared Spectrograph (IRS; Houck et al. 2004) on Spitzer to examine the behavior of a large sample of AGNs.

Important high-ionization lines accessible in the mid-IR include [Ne v] $(97.1 \mathrm{eV})$ at 14.32 and $24.32 \mu \mathrm{m}$, [O IV] $(54.9 \mathrm{eV})$ at $25.89 \mu \mathrm{m}$, and [Ne III] $(41 \mathrm{eV})$ at $15.56 \mu \mathrm{m}$. Because of their very high ionization potential, the $[\mathrm{Ne} \mathrm{v}]$ lines are considered to be reliable signposts for an AGN (Genzel et al. 1998; Armus et al. 2007). These lines have been used to estimate the accretion power in the local universe (Tommasin et al. 2010) and to identify low-luminosity AGNs in local galaxies (Satyapal et al. 2008; Goulding \& Alexander 2009). However, they are also produced in supernova remnants (SNRs; Oliva et al. 1999; Smith

\footnotetext{
* This work is based on observations made with the Spitzer Space Telescope, which is operated by the Jet Propulsion Laboratory, California Institute of Technology, under NASA contract 1407.

4 Associate Astronomer, Steward Observatory, University of Arizona, AZ 85721, USA.
}

et al. 2009), planetary nebulae (PNe; Pottasch et al. 2009), and Wolf-Rayet stars (W-R; Schaerer \& Stasińska 1999). Although the [Ne v] luminosities of these objects are low, $\sim 10^{34} \mathrm{erg} \mathrm{s}^{-1}$ (Smith et al. 2009; Pottasch et al. 2009), several thousands of them might also produce detectable [Ne v] emission.

Despite the lower ionization potential of [O IV], the $25.89 \mu \mathrm{m}$ emission line appears to be an accurate indicator of AGN power, since it correlates well with the hard $(>14 \mathrm{keV}) \mathrm{X}$-ray luminosity (Meléndez et al. 2008b; Rigby et al. 2009; DiamondStanic et al. 2009) and the mid-IR [Ne v] emission lines (Dudik et al. 2009; Weaver et al. 2010). However, this line also appears in the spectra of starburst galaxies with no other evidence for AGNs (Lutz et al. 1998a; Bernard-Salas et al. 2009), where it is attributed to W-R stars (Crowther et al. 1999; Schaerer \& Stasińska 1999) and/or to shocks (Allen et al. 2008; Lutz et al. 1998a).

The [Ne III] $15.56 \mu \mathrm{m}$ line is excited by young, massive stars (Verma et al. 2003; Brandl et al. 2006; Beirão et al. 2006, 2008; Ho \& Keto 2007; Bernard-Salas et al. 2009; AlonsoHerrero et al. 2009; Pereira-Santaella et al. 2010). Nonetheless, it seems to be a reasonably good proxy for AGN luminosity, at least for reasonably high luminosities (Gorjian et al. 2007; Dudik et al. 2009; Meléndez et al. 2008b; Tommasin et al. 2008). Quantifying when its excitation is dominated by an active nucleus would allow it to be used in concert with the other highionization lines to probe conditions in the neighborhoods of AGNs.

In this paper, we present a statistical study of the behavior of these lines in a sample of 426 galaxies. The data are compiled from the literature on high spectral resolution $(R \sim 600)$ IRS measurements, as well as our own new measurements of low-luminosity Seyfert galaxies and luminous infrared galaxies (LIRGs). We describe the sample and data reduction, 


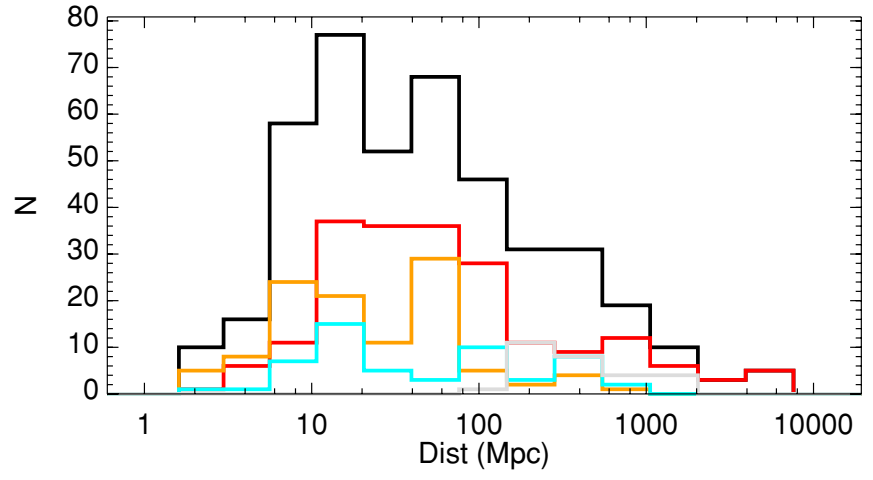

Figure 1. Distribution of the galaxy distances of the complete sample (black), QSO (gray), Seyfert 1 and 2 galaxies (red), LINERs (blue), and $\mathrm{H}$ II galaxies (orange).

(A color version of this figure is available in the online journal.)

respectively, in Sections 2 and 3. Section 4 explores the correlations among the high-ionization emission lines. In Section 5, we discuss the star formation (SF) contribution to these lines. Section 6 compares the SF in Seyfert 1 and 2 galaxies. Finally, in Section 7 we use photoionization models to study the physical conditions in the narrow-line regions (NLRs).

Throughout this paper, we assume a flat cosmology with $H_{0}=70 \mathrm{~km} \mathrm{~s}^{-1} \mathrm{Mpc}^{-1}, \Omega_{\mathrm{M}}=0.3$, and $\Omega_{\Lambda}=0.7$.

\section{THE SAMPLE}

The sample contains 426 galaxies (Table 1) for which high spectral resolution $(R \sim 600)$ Spitzer/IRS spectra were available, either in the literature (Weedman et al. 2005; Ogle et al. 2006; Farrah et al. 2007; Gorjian et al. 2007; Tommasin et al. 2008, 2010; Veilleux et al. 2009; Dale et al. 2009; BernardSalas et al. 2009; Goulding \& Alexander 2009; M. PereiraSantaella et al. 2011, in preparation), in the Spitzer archive, or observed through the programs 40936 and 50597 (PI: G. H. Rieke). AGN-type classifications were taken from NASA Extragalactic Database (NED). We designated only pure cases as type 2 and included within type 1 all intermediate cases (i.e., 1.2, 1.5, 1.8, 1.9) based on observations and modeling of the nuclear spectral energy distributions (Alonso-Herrero et al. 2003; Ramos Almeida et al. 2009). We could not find the nuclear activity classification for 32 of the galaxies. The sample includes 28 quasi-stellar objects (QSOs), 76 Seyfert 1, 125 Seyfert 2, 55 LINERs, and $110 \mathrm{H}$ II or starburst galaxies (see Table 2). The luminosity of the active galaxies ranges from QSO (QUEST sample; Veilleux et al. 2009) to typical Seyfert galaxies (12 $\mu \mathrm{m}$ sample; Rush et al. 1993; Tommasin et al. 2010), relatively lowluminosity Seyferts (RSA sample; Maiolino \& Rieke 1995; Ho et al. 1997; Diamond-Stanic et al. 2009), and LINERs (Sturm et al. 2006). According to their IR luminosities 71 sample members are classified as LIRGs $\left(L_{\mathrm{IR}}=10^{11}-10^{12} L_{\odot}\right)$ and 54 as ultraluminous infrared galaxies (ULIRGs; $L_{\mathrm{IR}}>10^{12} L_{\odot}$ ). We found in the Revised Bright Galaxy Sample catalog (Sanders et al. 2003; Surace et al. 2004) the IRAS fluxes for 196 of our galaxies which we used to calculate their $L_{\mathrm{IR}}$ as defined in Sanders \& Mirabel (1996).

Note that the LINER group includes very different galaxies. IR-bright LINERs $\left(L_{\mathrm{IR}} / L_{\mathrm{B}} \gtrsim 1\right)$ have IR spectral energy distributions similar to starbursts, although high-ionization lines and hard X-ray cores are detected in some, suggesting the presence of an AGN. In comparison, IR-faint LINERs $\left(L_{\mathrm{IR}} / L_{\mathrm{B}} \lesssim 1\right)$ seem to be powered by an AGN (Sturm et al. 2006; Satyapal et al. 2004).
Figure 1 shows the distance distribution of the sample. The median distance is $60 \mathrm{Mpc}$ and most $(68 \%)$ of the galaxies are between 15 and $400 \mathrm{Mpc}$.

\section{DATA REDUCTION}

In addition to results from the literature, we utilized the measurements for the 88 galaxies of the RSA sample observed with the high spectral resolution modules as well as for the 34 LIRGs of the Alonso-Herrero et al. (2006) sample (3 of these LIRGs are also members of the RSA sample). We retrieved the basic calibrated data (BCD) from the Spitzer archive processed by pipeline version S18.7. We subtracted the background contribution when a dedicated sky observation was available. Note however that the sky subtraction is not important to measure these fine structure emission lines, and it just improves the final quality of the spectra by removing bad pixels. Then, we extracted the spectra using the standard programs included in the Spitzer IRS Custom Extraction (SPICE) package provided by the Spitzer Science Center (SSC). We assumed point source calibration for all the galaxies, which is a good approximation for most of the galaxies, at least for the high-ionization lines. For the galaxies observed in the mapping mode we extracted the nuclear spectra from the data cubes using a square aperture of 13 . $4 \times 13$.". 4 and then we applied an aperture correction (M. Pereira-Santaella et al. 2011, in preparation). We used a Gaussian profile to fit the emission lines. For nine galaxies (NGC 777, NGC 3254, NGC 3486, NGC 3941, NGC 4138, NGC 4378, NGC 4472, NGC 4698, NGC 5631), we were not able to measure any spectral feature due to the low signal-tonoise ratio of the spectra. They are neither listed in Table 1 nor included in the analyzed sample.

Table 2 includes the number of detections of the $[\mathrm{Ne}$ v]14.32 $\mu \mathrm{m},[\mathrm{Ne} \mathrm{v}] 24.32 \mu \mathrm{m}$, and [O IV]25.89 $\mu \mathrm{m}$ emission lines, and in Table 3 we show the observed median line ratios for each galaxy type both for galaxies from the literature and those analyzed by us. Note that there are four detections of the $[\mathrm{NeV}] 14.32 \mu \mathrm{m}$ line and two of the $[\mathrm{NeV}] 24.32 \mu \mathrm{m}$ line in galaxies classified as H II. For these galaxies (NGC 613, NGC 1792, NGC 3621, and NGC 5734), the detection of the [Ne v] lines is the only evidence of AGN activity. We decided to keep their H II classification because (1) the AGN may be extremely obscured or of very low luminosity and, thus, the nuclear spectra might be dominated by SF features and (2) these lines are also detected in SNRs, PNe, and W-R stars which could be the origin of the [ $\mathrm{Ne} \mathrm{V}]$ emission in these galaxies.

Table 3 gives the median and deviation of all the line ratios we discuss in this paper for each type of galaxy. The line fluxes for all the galaxies are listed in Table 1.

\section{THE MID-IR HIGH-IONIZATION EMISSION LINES}

\subsection{The [O IV] Versus [Ne V] Correlations}

The $[\mathrm{Ne} \mathrm{v}] 24.32 \mu \mathrm{m}$ and the [O IV]25.89 $\mu \mathrm{m}$ emission lines are commonly detected in active galaxies (Lutz et al. 1998b; Genzel et al. 1998; Tommasin et al. 2010). The detection of the former is considered a good indicator of AGN activity because of its high ionization potential. However, using the [O IV] $25.89 \mu \mathrm{m}$ line as an AGN tracer is not as straightforward. For optically classified Seyfert galaxies the [O IV] $25.89 \mu \mathrm{m}$ luminosity is a good proxy for the AGN intrinsic luminosity (Diamond-Stanic et al. 2009; Meléndez et al. 2008b) and there is a good correlation between the $[\mathrm{Ne} \mathrm{v}] 24.32 \mu \mathrm{m}$ and the [O IV]25.89 $\mu \mathrm{m}$ emission 

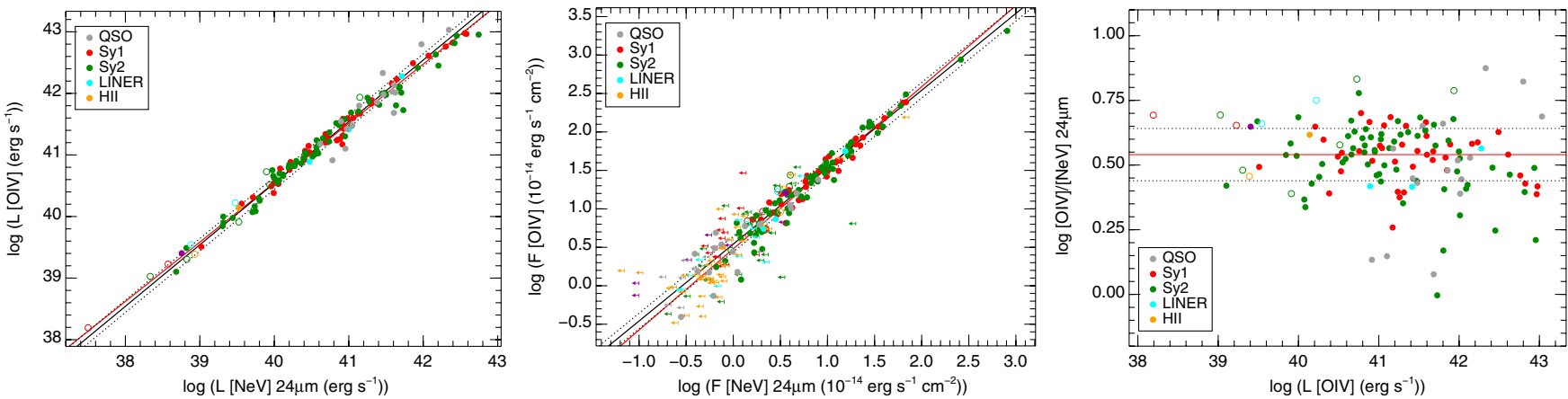

Figure 2. Relationship between the [O IV] $25.89 \mu \mathrm{m}$ and [Ne v]24.32 $\mu \mathrm{m}$ luminosities (left) and fluxes (middle). The red line is the best fit and the black line is the best linear fit. The dashed lines mark the $1 \sigma$ deviation. QSOs are plotted as gray circles, Sy1 as red circles, Sy2 as green circles, LINERs as blue circles, H iI galaxies as orange circles, and those galaxies without a classification as purple circles. The open symbols mark the galaxies with SF which may contribute to the [O IV] $25.89 \mu \mathrm{m}$ emission (see Section 4.2 and the right panel of Figure 4). The right panel shows the [O Iv] $25.89 \mu \mathrm{m} /[\mathrm{Ne} v] 24.32 \mu \mathrm{m}$ ratio vs. the [O Iv] $25.89 \mu \mathrm{m}$ luminosity. The solid red line is the ratio obtained from the linear fit and the dashed lines mark the $1 \sigma$ deviation.

(A color version of this figure is available in the online journal.)

Table 1

Sample and Mid-IR Emission Line Fluxes

\begin{tabular}{|c|c|c|c|c|c|c|c|c|c|c|}
\hline Name & $\begin{array}{c}\text { R.A. }^{\mathrm{a}} \\
(\mathrm{J} 2000.0)\end{array}$ & $\begin{array}{c}\text { Decl. }^{\mathrm{a}} \\
(\mathrm{J} 2000.0)\end{array}$ & $\begin{array}{l}\text { Dist. }^{b} \\
\text { (Mpc) }\end{array}$ & $\begin{array}{l}\text { Spect. } \\
\text { Class. }^{\text {a }}\end{array}$ & $\begin{array}{c}{[\mathrm{Ne} \mathrm{II}]} \\
12.81 \mu \mathrm{m}\end{array}$ & $\begin{array}{c}{[\mathrm{Ne} \mathrm{v}]} \\
14.32 \mu \mathrm{m}\end{array}$ & $\begin{array}{c}{[\mathrm{Ne} \text { III] }} \\
15.56 \mu \mathrm{m}\end{array}$ & $\begin{array}{c}{[\mathrm{Ne} \mathrm{v}]} \\
24.32 \mu \mathrm{m}\end{array}$ & $\begin{array}{c}{\left[\mathrm{O}_{\mathrm{IV}}\right]} \\
25.89 \mu \mathrm{m} \\
\end{array}$ & Ref. \\
\hline Mrk $335^{\mathrm{d}}$ & 000619.5 & +201210 & 113 & Sy1 & 0.25 & 0.38 & 0.61 & 2.0 & 7.2 & 4 \\
\hline NGC 23 & 000953.4 & +255525 & 64.5 & H II & 96 & $<0.50$ & 13 & $<0.44$ & 1.4 & 2 \\
\hline NGC 24 & 000956.5 & -245747 & 7.30 & $\ldots$ & 3.1 & $<0.88$ & 1.3 & $\ldots$ & $<0.42$ & 5 \\
\hline Mrk $938^{d}$ & 001106.5 & -120626 & 85.3 & Sy2 & 52 & $<2.2$ & 6.4 & $<0.37$ & $<0.66$ & 4 \\
\hline IRAS F00188-0856 & 002126.5 & -083926 & 600 & LINER & 4.7 & $<0.18$ & 0.69 & $<1.6$ & $<0.90$ & 3 \\
\hline IRAS 00198-7926 & 002153.6 & -791007 & 329 & Sy2 & 6.2 & 12 & 14 & 11 & 33 & 4 \\
\hline PG $0026+129$ & 002913.6 & +131603 & 672 & QSO & 0.23 & 0.47 & 0.79 & $<0.33$ & 2.1 & 1 \\
\hline ESO 012-G021 ${ }^{\mathrm{d}}$ & 004046.2 & -791424 & 144 & Sy1 & 12 & 3.2 & 6.4 & 4.6 & 16 & 4 \\
\hline IRAS F00397-1312 & 004215.5 & -125602 & 1329 & $\mathrm{H}_{\text {II }}$ & 4.4 & $<0.20$ & 2.7 & $<1.5$ & $<1.2$ & 3 \\
\hline NGC 253 & 004733.1 & -251717 & 2.50 & H II & 2832 & $<21$ & 205 & $<73$ & 155 & 8 \\
\hline
\end{tabular}

Notes. Fluxes are expressed in units of $10^{-14} \mathrm{erg} \mathrm{cm}^{-2} \mathrm{~s}^{-1}$.

${ }^{a}$ Coordinates and optical spectroscopic classification from NED.

${ }^{\mathrm{b}}$ We calculated the distance from the redshift assuming a flat cosmology with $H_{0}=70 \mathrm{~km} \mathrm{~s}^{-1} \mathrm{Mpc}^{-1}, \Omega_{\mathrm{M}}=0.3$, and $\Omega_{\Lambda}=0.7$, except for the galaxies from Dale et al. (2009), Bernard-Salas et al. (2009), and Goulding \& Alexander (2009) for which we used the distances adopted by these authors.

${ }^{c}$ Member of the RSA sample.

d Member of the $12 \mu \mathrm{m}$ sample.

References. (1) Veilleux et al. 2009; (2) this work; (3) Farrah et al. 2007; (4) Tommasin et al. 2008, 2010; (5) Dale et al. 2009; (6) Gorjian et al. 2007; (7) Goulding \& Alexander 2009; (8) Bernard-Salas et al. 2009.

(This table is available in its entirety in a machine-readable form in the online journal. A portion is shown here for guidance regarding its form and content.)

Table 2

The Sample

\begin{tabular}{lcccc}
\hline \hline \multicolumn{1}{c}{ Type } & $N$ & {$[\mathrm{Ne}$ v $] 14.3 \mu \mathrm{m}$} & {$[\mathrm{Ne}$ v $] 24.3 \mu \mathrm{m}$} & {$[\mathrm{O}$ IV $]$} \\
\hline QSO & 28 & 22 & 16 & 25 \\
Seyfert 1 & 76 & 59 & 42 & 63 \\
Seyfert 2 & 125 & 89 & 69 & 101 \\
LINER & 55 & 10 & 6 & 27 \\
H II/starburst & 110 & 4 & 2 & 59 \\
Unknown & 32 & 2 & 1 & 7 \\
Total & 426 & 186 & 136 & 282 \\
\hline
\end{tabular}

Notes. $N$ is the number of galaxies of each type. For each type, we give the number of detections of the $[\mathrm{Nev}] 14.32 \mu \mathrm{m},[\mathrm{Nev}] 24.32 \mu \mathrm{m}$, and [O IV $] 25.89 \mu \mathrm{m}$ emission lines.

in AGNs (Dudik et al. 2009; Weaver et al. 2010). However, the [O IV] $25.89 \mu \mathrm{m}$ line is also produced by W-R stars (Schaerer \& Stasińska 1999) and is observed in starburst galaxies with no other evidence for the presence of an AGN (Lutz et al. 1998a; Bernard-Salas et al. 2009).
The left panel of Figure 2 shows the tight correlation between the $[\mathrm{Ne} \mathrm{v}] 24.32 \mu \mathrm{m}$ and [O IV] $25.89 \mu \mathrm{m}$ lines spanning at least five orders of magnitude in luminosity $\left(38<\log L_{\text {[О гv] }}\right.$ $\left(\right.$ erg $\left.\left.\mathrm{s}^{-1}\right)<43\right)$. We do not find any dependence between the optical classification of the nuclear activity and this correlation. This implies that the ionization parameter is similar in type 1 and 2 Seyfert galaxies (see Section 7). The slope of the best fit to the luminosity data is $0.96 \pm 0.02$ with a 0.14 dex dispersion. It is reasonable to assume a linear correlation between these two emission lines, and this yields a ratio $\left[\mathrm{O}_{\mathrm{IV}}\right] 25.89 \mu \mathrm{m} /[\mathrm{NeV}] 24.32 \mu \mathrm{m}=3.5$, with rms scatter of 0.8 .

The $[\mathrm{Ne} \mathrm{v}] 24.32 \mu \mathrm{m}$ line is detected in $\sim 50 \%$ of the optically classified Seyfert galaxies (see Table 2). However, for a considerable number of galaxies we have the [O IV] $25.89 \mu \mathrm{m}$ measurement and an upper limit for the $[\mathrm{Ne} \mathrm{v}] 24.32 \mu \mathrm{m}$ flux. We plot these fluxes and upper limits (middle panel of Figure 2) for all the galaxies. The upper limits to the [Ne v]24.32 $\mu \mathrm{m}$ flux are compatible with the correlation for most of the Seyfert galaxies. However, the $[\mathrm{O} \mathrm{IV}] 25.89 \mu \mathrm{m}$ line is detected in more 

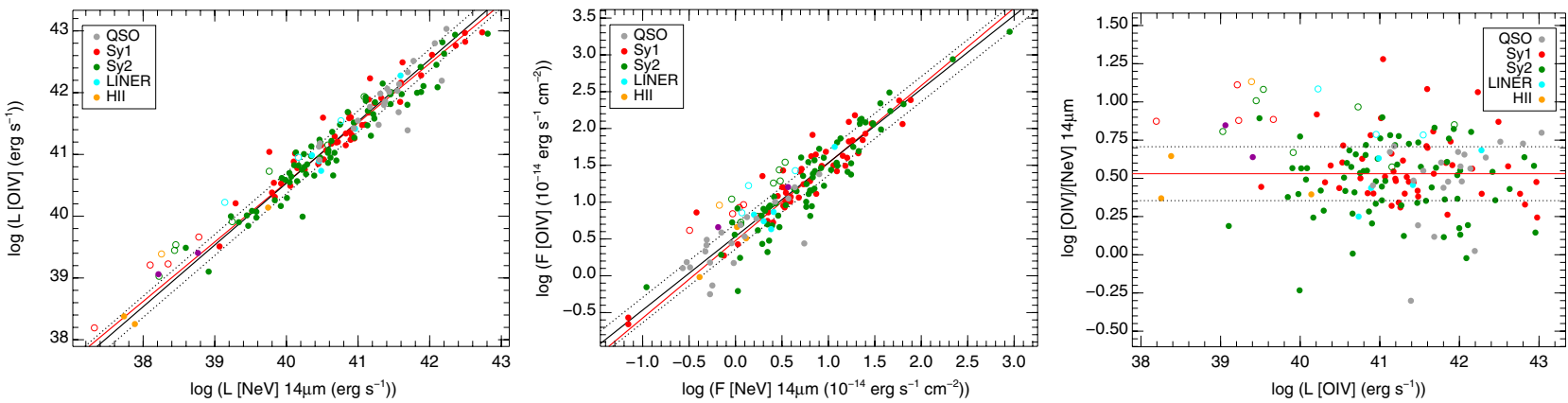

Figure 3. Relationship between the [O IV $] 25.89 \mu \mathrm{m}$ and $[\mathrm{Nev}] 14.32 \mu \mathrm{m}$ luminosities (left) and fluxes (middle). The right panel shows the [O Iv $] 25.89 \mu \mathrm{m} /$ $[\mathrm{Ne}$ v]14.32 $\mu \mathrm{m}$ ratio vs. the [O Iv]25.89 $\mu \mathrm{m}$ luminosity. Symbols are as in Figure 2.

(A color version of this figure is available in the online journal.)
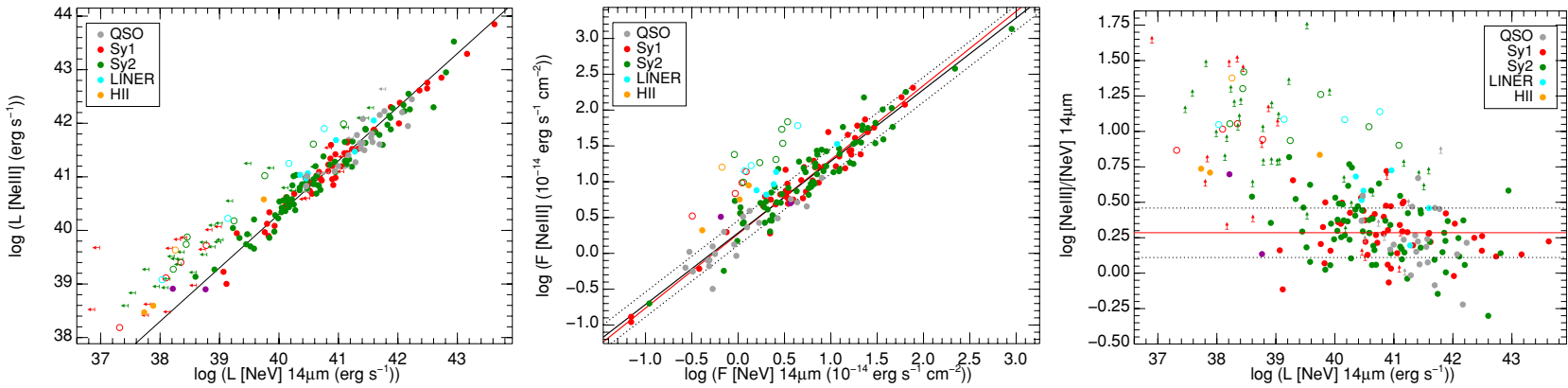

Figure 4. Relation between the [Ne III] $15.56 \mu \mathrm{m}$ and [Ne v]14.32 $\mu \mathrm{m}$ emission. Galaxy symbols are as in Figure 2. The open symbols mark those galaxies $3 \sigma$ above the $[\mathrm{Ne}$ V]14.32 $\mu \mathrm{m}$ vs. [Ne III] $15.56 \mu \mathrm{m}$ flux correlation. The black line in the left panel is the ratio obtained from the flux correlation. In the left panel only the $[\mathrm{Ne}$ v $24.32 \mu \mathrm{m}$ upper limits of the QSOs and Seyfert galaxies are included.

(A color version of this figure is available in the online journal.)

than $50 \%$ of the H II galaxies in our sample. In $90 \%$ of these galaxies, the sensitivity of the $[\mathrm{Ne} \mathrm{v}] 24.32 \mu \mathrm{m}$ measurement (or upper limits) is inadequate to probe whether the [O IV] emission is associated with a hidden AGN.

In short, we find that the [O IV] $25.89 \mu \mathrm{m}$ luminosity is well correlated with the intrinsic AGN luminosity (i.e., the [Ne v] luminosity) for Seyfert galaxies and quasars with $L_{[\mathrm{O} \text { Iv] }}>$ $10^{39} \mathrm{erg} \mathrm{s}^{-1}$ to, at least, $L_{[\mathrm{O} \text { Iv }]} \sim 10^{43} \mathrm{erg} \mathrm{s}^{-1}$. For lower luminosities the fraction of the [O IV] $25.89 \mu \mathrm{m}$ emission produced by SF may be considerable. Thus, the [O Iv]25.89 $\mu \mathrm{m}$ luminosity may not be an accurate tracer of the AGN luminosity for galaxies with $L_{[\mathrm{O} \text { Iv }]}<10^{39} \mathrm{erg} \mathrm{s}^{-1}$ (Goulding \& Alexander 2009).

The linear correlation also holds for the $[\mathrm{Ne} \mathrm{v}] 14.32 \mu \mathrm{m}$ and the [O Iv]25.89 $\mu \mathrm{m}$ luminosities (Figure 3), although the dispersion is larger ([O IV $] 25.89 \mu \mathrm{m} /[\mathrm{Ne} \mathrm{v}] 14.32 \mu \mathrm{m}=3.4$, with rms scatter of 1.4 assuming a linear fit).

\subsection{The [Ne III] and [Ne II] Emission Lines}

Due to the intermediate ionization potential of $\mathrm{Ne}^{+2}(41.0 \mathrm{eV})$, the [Ne III] $15.56 \mu \mathrm{m}$ emission line may be produced by young stars or by AGNs. This line is observed in star-forming galaxies (Verma et al. 2003; Brandl et al. 2006; Beirão et al. 2006, 2008; Ho \& Keto 2007; Bernard-Salas et al. 2009; Dale et al. 2009; Pereira-Santaella et al. 2010), and is also correlated with the AGN intrinsic luminosity (Gorjian et al. 2007; Deo et al. 2007; Tommasin et al. 2008; Meléndez et al. 2008a).

The middle panel of Figure 4 shows the relation between the observed flux of the [Ne III] $15.56 \mu \mathrm{m}$ and [Ne v] $14.32 \mu \mathrm{m}$ emission lines for our sample of AGNs and for the $4 \mathrm{H}$ II galaxies with $[\mathrm{Ne} v]$ detections. We compare these lines because they are close in wavelength and the effect of differ- ential extinction is minimized. It is clear that some galaxies have an excess of [Ne III] $15.56 \mu \mathrm{m}$ emission relative to that of the $[\mathrm{Nev}] 14.32 \mu \mathrm{m}$, which we attribute to SF (see Section 5). Therefore, to obtain the fit to the data we use the outliers resistant linear fit algorithm provided by the IDL function ROBUST_LINEFIT. The slope of this fit is $1.02 \pm 0.02$ with a scatter of about 0.2 dex around the fit. The result is consistent with a simple correlation; by fixing the slope to unity, we obtain the ratio [Ne III] $15.56 \mu \mathrm{m} /[\mathrm{Ne} \mathrm{v}] 14.32 \mu \mathrm{m}=1.9$, with rms scatter of 0.8 . This correlation was previously reported by Gorjian et al. (2007), although they found a slight dependence of the ratio with the [Ne v] $14.32 \mu \mathrm{m}$ luminosity (slope 0.89). Their sample included $53 \mathrm{X}$-ray selected AGNs with [Ne v] $14.32 \mu \mathrm{m}$ luminosities between $10^{39}$ and $10^{43} \mathrm{erg} \mathrm{s}^{-1}$. The larger number of galaxies in our sample allows us to minimize the contribution of the galaxies contaminated by SF to the fit and the luminosity dependence of the correlation is substantially reduced. Where the AGN clearly dominates, the points fall around the line determined for the whole sample by ROBUST_LINEFIT, thus supporting our interpretation that this fit determines the relationship for AGNs in general.

The $[\mathrm{Ne}$ II] $12.81 \mu \mathrm{m}$ emission traces young $(<10 \mathrm{Myr}) \mathrm{SF}$ (Roche et al. 1991; Thornley et al. 2000; Verma et al. 2003; Rigby \& Rieke 2004; Snijders et al. 2007; Ho \& Keto 2007; Díaz-Santos et al. 2010). The ionization potential of this emission line is $21 \mathrm{eV}$ and thus it is mainly produced in H II regions. Figure 5 shows that the scatter in the $[\mathrm{Ne}$ II] $12.81 \mu \mathrm{m} /$ $[\mathrm{Nev}] 14.32 \mu \mathrm{m}$ ratio $(0.7 \mathrm{dex})$ is larger than that found in the other line ratios; for the lower AGN luminosities there is a large scatter. There is a weak trend of [Ne II] $12.81 \mu \mathrm{m} /$ $[\mathrm{Ne} v] 14.32 \mu \mathrm{m}$ ratios decreasing with increasing AGN luminosity. Assuming that there is an intrinsic ratio for AGN, this behavior implies that the AGN does not dominate the 


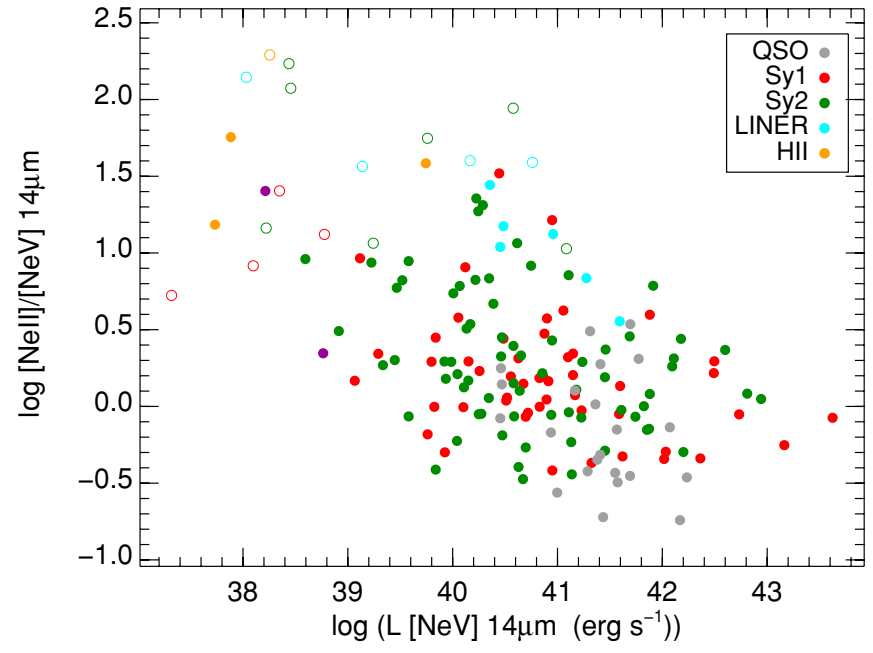

Figure 5. [Ne II] $12.81 \mu \mathrm{m} /[\mathrm{Ne}$ v]14.32 $\mu \mathrm{m}$ ratio vs. [Ne v]14.32 $\mu \mathrm{m}$ luminosity. Symbols are as in Figure 4.

(A color version of this figure is available in the online journal.)

[Ne II]12.81 $\mu \mathrm{m}$ output and that there is a wide range of star formation rates (SFR) contributing to its luminosity. However, for the most luminous objects $\left(\log L_{[\mathrm{Nev}] 14 \mu \mathrm{m}}\left(\mathrm{erg} \mathrm{s}^{-1}\right)>41.5\right)$, the smaller scatter indicates that the relative contribution of the AGN to the total $[\mathrm{Ne} \mathrm{II}] 12.81 \mu \mathrm{m}$ excitation is higher.

Sturm et al. (2002) determined that the pure AGN $[\mathrm{Ne} \mathrm{v}] 14.32 \mu \mathrm{m} /[\mathrm{Ne} \mathrm{II}] 12.81 \mu \mathrm{m}$ ratio is 1.1 , but we find many Seyfert galaxies with higher ratios. The largest [Ne v] $14.32 \mu \mathrm{m} /$ [Ne II]12.81 $\mu \mathrm{m}$ ratio in our sample is $\sim 5.5$, which is compatible with the largest ratio predicted by NLR photoionization models (Groves et al. 2006, their Figure 11). With our data (Figure 5) we are not able to determine the pure AGN ratio, which also depends on the ionization parameter (Groves et al. 2006), but it is likely that it is between that found by Sturm et al. (2002) and the largest ratio in our sample.

The [O IV] $25.89 \mu \mathrm{m} /[\mathrm{Ne}$ II $] 12.81 \mu \mathrm{m}$ ratio can be used to separate AGNs from star-forming galaxies (Genzel et al. 1998; Sturm et al. 2002; Peeters et al. 2004; Dale et al. 2009). We plot this ratio versus the [O IV] $25.89 \mu$ m luminosity in Figure 6. Most of the active galaxies have ratios larger than 0.35 whereas the ratios for $\mathrm{H}$ II galaxies are lower than 0.05 (Figure 5 of Dale et al. 2009). LINERs appear in the region between these ratios (Figure 6). A considerable number of $\mathrm{H}$ II galaxies also have $\left[\mathrm{O}_{\mathrm{IV}}\right] 25.89 \mu \mathrm{m} /[\mathrm{Ne} \mathrm{II}] 12.81 \mu \mathrm{m}$ ratios above 0.05 . The [Ne v] lines are not detected for them although their upper limits are compatible with the [O IV] $25.89 \mu \mathrm{m}$ versus [Ne v] correlations and thus a low-luminosity AGN could be the origin of the [O IV] $25.89 \mu \mathrm{m}$ emission. The large scatter in the [O IV] $25.89 \mu \mathrm{m} /[\mathrm{Ne}$ II] $12.81 \mu \mathrm{m}$ ratio, due to the different SFRs in the AGNs, does not allow us to determine the value of the pure AGN ratio. The largest [O IV] $25.89 \mu \mathrm{m} /[\mathrm{Ne}$ II] $12.81 \mu \mathrm{m}$ ratios that we find in AGNs are $\sim 10$.

\section{STAR FORMATION CONTRIBUTIONS TO THE MID-IR HIGH-IONIZATION EMISSION LINES}

\section{1. [O IV] Contamination by Star Formation}

In Figures 2 and 3, there are few outliers above the [O Iv]25.89 $\mu \mathrm{m}$ versus [Nev] correlations. Most of them are represented by open symbols, which indicate that their $[\mathrm{Ne}$ III $] 15.56 \mu \mathrm{m} /[\mathrm{Ne} \mathrm{V}] 14.32 \mu \mathrm{m}$ ratios (see Section 5.2) are larger than those found in AGN-dominated galaxies (QSO,

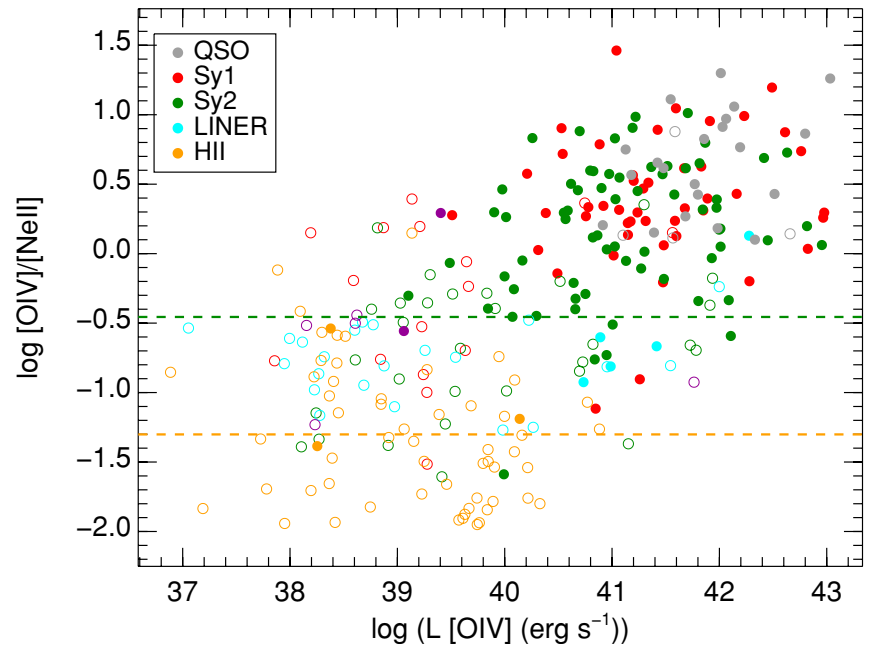

Figure 6. [O IV] $25.89 \mu \mathrm{m} /[\mathrm{Ne}$ II $] 12.81 \mu \mathrm{m}$ ratio vs. [O IV] $25.89 \mu \mathrm{m}$ luminosity. Galaxy symbols are as in Figure 2 . The dashed orange ([O IV] $/[\mathrm{Ne}$ II $]=0.05$ ) and green $([\mathrm{O} I V] /[\mathrm{Ne} I \mathrm{II}]=0.35)$ lines are the boundaries of the $\mathrm{H}$ II and Seyfert galaxies, respectively.

(A color version of this figure is available in the online journal.)

Sy1, and Sy2). It is likely that the [O IV]25.89 $\mu \mathrm{m}$ emission of these galaxies is contaminated by SF. We now compare the [O IV]25.89 $\mu \mathrm{m}$ emission of star-forming regions and AGNs. The [O IV] $25.89 \mu \mathrm{m} /[\mathrm{Ne}$ II] $12.81 \mu \mathrm{m}$ ratio in star-forming regions is $<0.05$ (Section 4.2). Taking this upper limit and using the relation between the $[\mathrm{Ne}$ II $] 12.81 \mu \mathrm{m}$ luminosity and the total IR $(8-1000 \mu \mathrm{m})$ luminosity $\left(L_{\mathrm{IR}}\right)$ from Ho \& Keto (2007) we obtain the following relation for star-forming galaxies:

$$
\log L_{[\mathrm{O} \text { IV }]}\left(\mathrm{erg} \mathrm{s}^{-1}\right)<\log L_{\mathrm{IR}}\left(\mathrm{erg} \mathrm{s}^{-1}\right)-4.7 \pm 0.6 .
$$

Rigby et al. (2009) calculated the ratio between the total AGN luminosity and the [O IV] $25.89 \mu \mathrm{m}$ emission. We use the ratio for type 1 AGNs $(\sim 2500)$ because the hard X-ray $(E>10 \mathrm{keV})$ emission used for the bolometric corrections may be affected by extinction in Seyfert 2's (Rigby et al. 2009):

$$
\log L_{\left[\mathrm{O}_{\text {IV }}\right.}\left(\mathrm{erg} \mathrm{s}^{-1}\right)=\log L_{\mathrm{AGN}}\left(\mathrm{erg} \mathrm{s}^{-1}\right)-3.4 \pm 0.4 .
$$

Then, combining both relations we find that the luminosity due to SF, $L_{\mathrm{IR}}$, has to be at least 20 times brighter than the AGN luminosity (i.e., AGN contribution to the total luminosity below $5 \%$ ) for the SF to dominate the [O IV]25.89 $\mu \mathrm{m}$ emission.

We note that the number of $\mathrm{H}$ II galaxies falls rapidly above $\log L_{\text {[O IV] }}\left(\mathrm{erg} \mathrm{s}^{-1}\right)=40.2$ (see Figure 6). Assuming that SF dominates the [O IV] $25.89 \mu \mathrm{m}$ emission at this luminosity in these galaxies, the equivalent IR luminosity $\left(L_{\mathrm{IR}}\right)$ would be $2 \times$ $10^{11} L_{\odot}$. This value of the $L_{\mathrm{IR}}$ seems reasonable since most of the $\mathrm{H}$ II galaxies with $\log L_{[\mathrm{O} \text { Iv] }}\left(\mathrm{erg} \mathrm{s}^{-1}\right)$ between 39.6 and 41.0 are classified as LIRGs. The $L_{\mathrm{IR}}$ limit calculated above is one order of magnitude larger than $L_{\star}$ (Takeuchi et al. 2003) so the reason for the lower number of $\mathrm{H}$ II galaxies above this [O IV] $25.89 \mu \mathrm{m}$ luminosity can be that the space density of luminous starforming galaxies decreases rapidly with increasing luminosity.

Out of 196 galaxies with estimates for $L_{\mathrm{IR}}$, [O IV] $25.89 \mu \mathrm{m}$ is detected in 122 . Only $2 \% \pm 2 \%$ of the $\mathrm{H}$ II galaxies have [O IV] $25.89 \mu \mathrm{m}$ luminosities $1 \sigma$ above those expected from their $L_{\mathrm{IR}}$. Likewise, SF can explain the observed [O IV] $25.89 \mu \mathrm{m}$ luminosities for $9 \% \pm 4 \%$ of the Seyfert galaxies (Figure 7). Thus, the [O IV] $25.89 \mu \mathrm{m}$ emission of Seyferts is generally dominated by the AGN, whereas for optically classified H II 
Table 3

Median Line Ratios

\begin{tabular}{|c|c|c|c|c|c|}
\hline \multirow[t]{2}{*}{ Ratio } & \multicolumn{5}{|c|}{ Median Ratio } \\
\hline & QSO & Sy1 & Sy2 & LINER & $\mathrm{H}_{\text {II }}$ \\
\hline$[\mathrm{O}$ Iv $] 25.89 \mu \mathrm{m} /[\mathrm{Ne} \mathrm{v}] 24.32 \mu \mathrm{m}$ & $3.3 \pm 1.2^{\mathrm{a}}$ & $3.6 \pm 0.6$ & $3.5 \pm 0.6$ & $3.7 \pm 1.0^{\mathrm{a}}$ & $4.1 \pm 1.3^{\mathrm{a}}$ \\
\hline$\left[\mathrm{O}_{\mathrm{IV}}\right] 25.89 \mu \mathrm{m} /[\mathrm{Ne} \mathrm{v}] 14.32 \mu \mathrm{m}$ & $3.6 \pm 0.9$ & $3.5 \pm 1.0$ & $3.5 \pm 1.3$ & $4.8 \pm 2.0^{\mathrm{a}}$ & $4.4 \pm 2.0^{\mathrm{a}}$ \\
\hline$[\mathrm{Ne} \mathrm{v}] 24.32 \mu \mathrm{m} /[\mathrm{Ne} \mathrm{v}] 14.32 \mu \mathrm{m}$ & $1.0 \pm 0.3^{\mathrm{a}}$ & $1.1 \pm 0.3$ & $1.0 \pm 0.3$ & $1.3 \pm 0.3^{\mathrm{a}}$ & $4.7 \pm 4.1^{\mathrm{a}}$ \\
\hline$[\mathrm{Ne}$ III $] 15.56 \mu \mathrm{m} /[\mathrm{Ne} \mathrm{v}] 14.32 \mu \mathrm{m}$ & $1.7 \pm 0.5$ & $1.9 \pm 0.6$ & $2.0 \pm 0.7$ & $5.3 \pm 3.7^{\mathrm{a}}$ & $6.8 \pm 1.7^{\mathrm{a}}$ \\
\hline$[\mathrm{Ne}$ II $] 12.81 \mu \mathrm{m} /[\mathrm{Ne} \mathrm{v}] 14.32 \mu \mathrm{m}$ & $0.7 \pm 0.4$ & $1.6 \pm 0.7$ & $2.0 \pm 1.1$ & $28 \pm 14^{\mathrm{a}}$ & $57 \pm 42^{\mathrm{a}}$ \\
\hline$[\mathrm{Ne}$ III $] 15.56 \mu \mathrm{m} /\left[\mathrm{O}_{\mathrm{IV}}\right] 25.89 \mu \mathrm{m}$ & $0.5 \pm 0.2$ & $0.6 \pm 0.2$ & $0.9 \pm 0.4$ & $2.3 \pm 1.1$ & $4.2 \pm 2.4$ \\
\hline$[\mathrm{Ne}$ II $] 12.81 \mu \mathrm{m} /[\mathrm{O}$ IV $] 25.89 \mu \mathrm{m}$ & $0.2 \pm 0.1$ & $0.5 \pm 0.3$ & $1.1 \pm 0.9$ & $6 \pm 2$ & $22 \pm 16$ \\
\hline$[\mathrm{Ne}$ III $] 15.56 \mu \mathrm{m} /[\mathrm{Ne} \mathrm{II}] 12.81 \mu \mathrm{m}$ & $2.0 \pm 1.0$ & $1.2 \pm 0.6$ & $0.8 \pm 0.4$ & $0.3 \pm 0.2$ & $0.17 \pm 0.07$ \\
\hline
\end{tabular}

Notes. Median ratios and uncertainties for each type of galaxies. The uncertainty is calculated as the median absolute deviation (should be multiplied by 1.48 to obtain the standard deviation).

a These values are calculated with less than 20 galaxies.

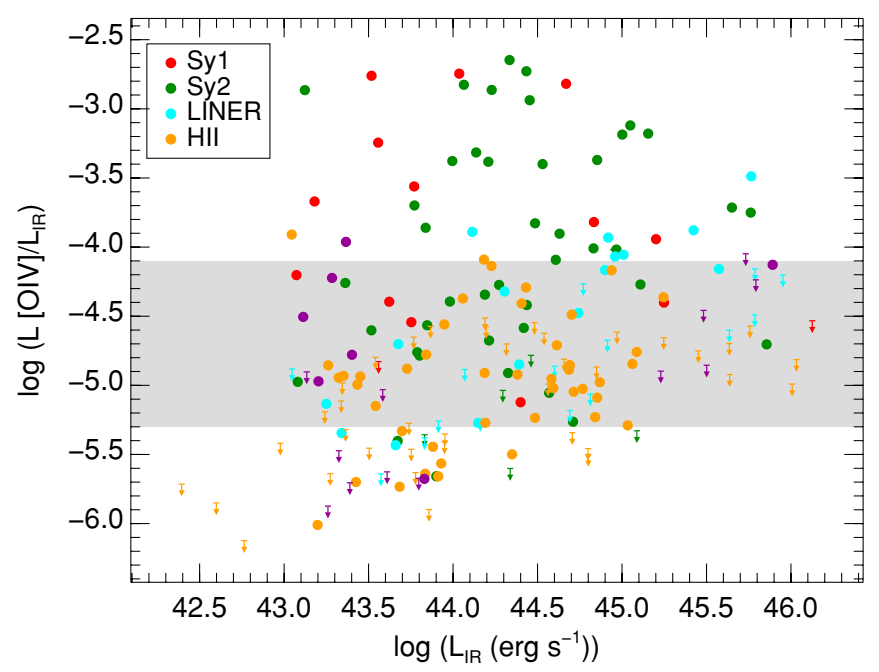

Figure 7. [O IV] $25.89 \mu \mathrm{m} / L_{\mathrm{IR}}$ ratio vs. $L_{\mathrm{IR}}$ luminosity. Galaxy symbols are as in Figure 2. The shaded region is the upper limit to the [O IV $] 25.89 \mu \mathrm{m} / L_{\mathrm{IR}}$ ratio of star-forming galaxies. Galaxies above this region need an AGN contribution to the [O IV] $25.89 \mu \mathrm{m}$ emission in order to explain the observed ratio.

(A color version of this figure is available in the online journal.)

galaxies SF is likely to be the origin of the [O IV $] 25.89 \mu \mathrm{m}$ emission.

\subsection{The [Ne III] Excess}

The $[\mathrm{Ne}$ III]15.56 $\mu \mathrm{m}$ emission may be produced by SF and/or AGN. In this section, we try to quantify the contribution of each one to the total [Ne III]15.56 $\mu \mathrm{m}$ output. For this purpose we used the correlations between [Ne v]14.32 $\mu \mathrm{m}$, [O IV]25.89 $\mu \mathrm{m}$, and [Ne III] $15.56 \mu \mathrm{m}$ (see Section 4). Combining the typical [O IV $] 25.89 \mu \mathrm{m} /[\mathrm{Nev}] 14.32 \mu \mathrm{m}$ and $[\mathrm{Ne}$ III] $15.56 \mu \mathrm{m} /[\mathrm{Ne} \mathrm{v}] 14.32 \mu \mathrm{m}$ ratios found in AGNs we estimate a ratio $[\mathrm{Ne}$ III $] 15.56 \mu \mathrm{m} /[\mathrm{O}$ IV $] 25.89 \mu \mathrm{m}=0.6$, with rms scatter of 0.3 for AGNs, ${ }^{5}$ or $\log [\mathrm{Ne}$ III $] /[\mathrm{O}$ IV $]=-0.22$. This estimated ratio is very similar to that obtained using a linear fit between the [Ne III] $15.56 \mu \mathrm{m}$ and [O IV] $25.89 \mu \mathrm{m}$ luminosities (Table 3). The median of this ratio for $\mathrm{H}$ II galaxies is 4.7, or $\log [\mathrm{Ne} \mathrm{III}] /[\mathrm{O} \mathrm{IV}]=0.67$, which is $\sim 8$ times larger than that estimated for active galaxies. Figure 8 shows that the observed ratio for Seyfert galaxies and QSOs falls around the predicted value for AGNs. Only a small fraction (4\%) of the H II galaxies

\footnotetext{
5 In this section, the term "AGN" refers to galaxies classified as QSO or Seyfert.
}

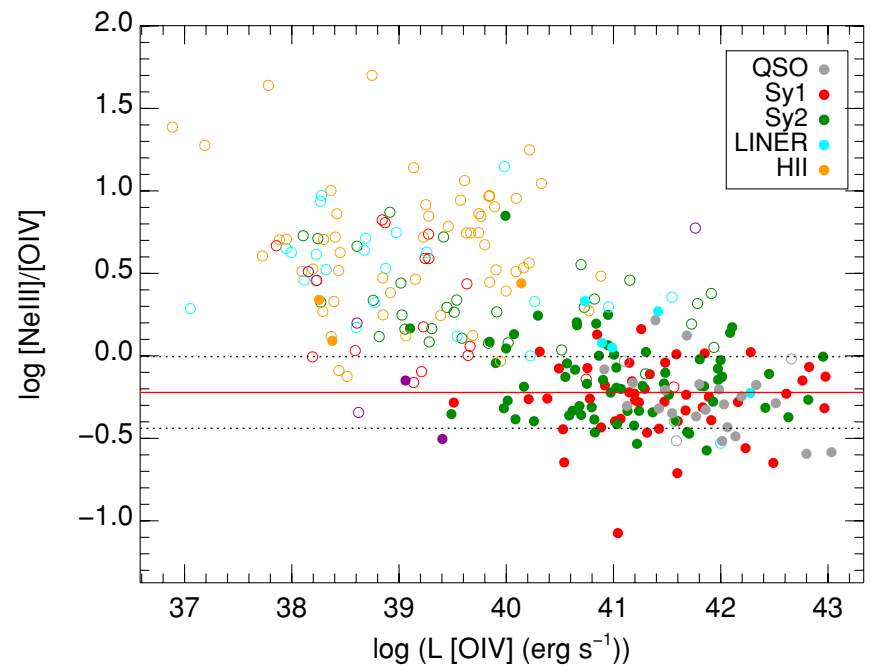

Figure 8. [Ne III] $15.56 \mu \mathrm{m} /[\mathrm{O}$ IV $] 25.89 \mu \mathrm{m}$ ratio vs. [O IV $] 25.89 \mu \mathrm{m}$ luminosity. Galaxy symbols are as in Figure 2. The solid red line is the [O IV] $25.89 \mu \mathrm{m} /$ $[\mathrm{Ne}$ III] $15.56 \mu \mathrm{m}$ ratio calculated from the linear fit to the [O IV] $25.89 \mu \mathrm{m}$ vs. $[\mathrm{Ne}$ III]15.56 $\mu \mathrm{m}$ luminosities. The dotted black lines are the $1 \sigma$ deviation.

(A color version of this figure is available in the online journal.)

have $[\mathrm{Ne}$ III] $15.56 \mu \mathrm{m} /[\mathrm{O}$ IV $] 25.89 \mu \mathrm{m}$ ratios in the AGN range. It is also apparent that $\mathrm{H}$ II galaxies, a large fraction of LINERs, and some Seyfert galaxies have an excess of [Ne III] $15.56 \mu \mathrm{m}$ relative to their [O IV] $25.89 \mu \mathrm{m}$ emission which can also be attributed to SF.

We find that the four HII galaxies with $[\mathrm{NeV}] 14.32 \mu \mathrm{m}$ detections have the $[\mathrm{Ne}$ III] $15.56 \mu \mathrm{m} /[\mathrm{Ne} \mathrm{V}] 14.32 \mu \mathrm{m}$ and $[\mathrm{Ne}$ II $] 12.81 \mu \mathrm{m} /[\mathrm{Ne} \mathrm{v}] 14.32 \mu \mathrm{m}$ ratios much larger than those of Seyfert galaxies (Table 3). This indicates that the SF contribution is larger than that in Seyfert galaxies. Thus, as suggested in Section 2 the AGN does not dominate the nuclear spectra of these galaxies.

As can be seen in the left panel of Figure 4, most of the outliers (galaxies more than $3 \sigma$ above the $[\mathrm{Nev}] 14.32 \mu \mathrm{m}$ versus [Ne III]15.56 $\mu \mathrm{m}$ flux correlation) have $L_{\text {[Ne III] }}<$ $10^{42} \mathrm{erg} \mathrm{s}^{-1}$. That is, for the most luminous objects the AGN dominates the $[\mathrm{Ne}$ III] $15.56 \mu \mathrm{m}$ emission. Using the relation by Ho \& Keto (2007) that relates SFR and the luminosity of the $[\mathrm{Ne}$ II $] 12.81 \mu \mathrm{m}$ and [Ne III] $15.56 \mu \mathrm{m}$ emission lines and assuming a $[\mathrm{Ne}$ III $] 15.56 \mu \mathrm{m} /[\mathrm{Ne}$ II] $12.81 \mu \mathrm{m}$ ratio $=0.3$ (see Section 5.3) we find that the SFR of the outliers is between 0.1 and $100 M_{\odot} \mathrm{yr}^{-1}$. 


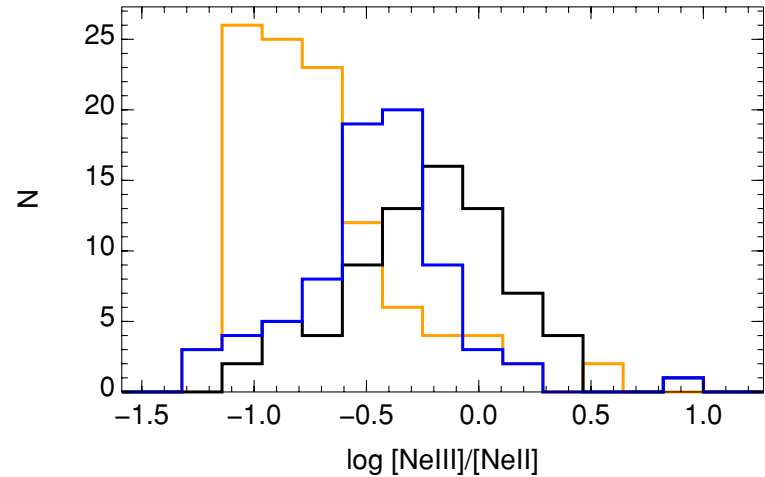

Figure 9. Distribution of the $[\mathrm{Ne} \mathrm{III}] 15.56 \mu \mathrm{m} /[\mathrm{Ne} \mathrm{II}] 12.81 \mu \mathrm{m}$ ratio. The orange histogram corresponds to the observed ratios in H II galaxies, black corresponds to the observed ratios in active galaxies with SF ([Ne III] $15.56 \mu \mathrm{m}$ / [O IV] $25.89 \mu \mathrm{m}$ ratios $>0.9$ ). The blue histogram shows the ratios calculated for Seyfert galaxies after subtracting the AGN contribution to the [Ne III] $15.56 \mu \mathrm{m}$ emission.

(A color version of this figure is available in the online journal.)

\subsection{The [Ne III]15.56 sp; $\mu \mathrm{m} /[\mathrm{Ne}$ II $] 12.81 \mu \mathrm{m}$ Ratio}

The $[\mathrm{Ne}$ III] $15.56 \mu \mathrm{m} /[\mathrm{Ne}$ II $] 12.81 \mu \mathrm{m}$ ratio traces the hardness of the radiation field and the age of the stellar population (Rigby \& Rieke 2004; Snijders et al. 2007). Figure 9 compares the $[\mathrm{Ne}$ III] $15.56 \mu \mathrm{m} /[\mathrm{Ne}$ II] $12.81 \mu \mathrm{m}$ ratio observed in $\mathrm{H}$ II galaxies (median 0.2) with that observed in Seyfert galaxies with $\mathrm{SF}^{6}$ (median 0.6). The larger ratio found in Seyfert galaxies can be explained if the AGN contributes noticeably to the total $[\mathrm{Ne}$ III $] 15.56 \mu \mathrm{m}$ emission but not to the [Ne II] $12.81 \mu \mathrm{m}$ emission (see Section 4.2). By assuming this we calculate the SF $[\mathrm{Ne}$ III] $15.56 \mu \mathrm{m} /[\mathrm{Ne}$ II] $12.81 \mu \mathrm{m}$ ratio in these Seyfert galaxies using the non-AGN [Ne III]15.56 $\mu \mathrm{m}$ emission that we estimated using the method described in Section 6. The median of this ratio becomes $\sim 0.3$, which is somewhat larger than that found in pure H II galaxies but it is in the range of the observed ratio in this class of galaxies (Brandl et al. 2006).

\section{COMPARISON OF STAR FORMATION IN SEYFERT 1 AND 2 GALAXIES}

The AGN unification scenario predicts that there should be no differences between the SF activity in Seyfert 1 and 2 galaxies. A number of studies compared the SFRs in Seyfert 1 and 2 galaxies. Some of them found enhanced SF activity in Seyfert 2 with respect Seyfert 1 galaxies for a given AGN luminosity (Maiolino et al. 1995; Buchanan et al. 2006; Deo et al. 2007; Meléndez et al. 2008a), whereas others did not find any differences between the two types (Kauffmann et al. 2003; Imanishi \& Wada 2004). However, as discussed by Shi et al. (2009), the different methods used to study the SF activity are sensitive to different stellar age ranges. Therefore, these apparently contradictory results may be consistent with each other. The [Ne III] $15.56 \mu \mathrm{m}$ line is sensitive to young ( $<5 \mathrm{Myr}$ ) massive SF (Rigby \& Rieke 2004; Snijders et al. 2007). Studies using this line (and the [Ne II]12.81 $\mu \mathrm{m}$ line) found that SF is enhanced in Seyfert 2 galaxies (Deo et al. 2007; Meléndez et al. 2008a). Kauffmann et al. (2003) did not find differences between Seyfert 1 and Seyfert 2 SF, but their method (the $4000 \AA$ break and the $\mathrm{H} \delta$ stellar absorption) was sensitive to stellar populations older than $100 \mathrm{Myr}$ (Shi et al. 2009). The same applies to the

\footnotetext{
6 Those with excess [Ne III] $15.56 \mu \mathrm{m}$.
}

PAH features used by Imanishi \& Wada (2004), which can be affected by the presence of the AGN (Diamond-Stanic \& Rieke 2010 and references therein).

It is also important to use the appropriate indicator for the AGN luminosity. We will use the [O IV]25.89 $\mu$ m emission, as it is a good isotropic indicator (Rigby et al. 2009; Diamond-Stanic et al. 2009), as long as the AGN dominates the [O IV] $25.89 \mu \mathrm{m}$ emission as we showed in Section 5.1.

We estimate the SF contribution to the [Ne III] $15.56 \mu \mathrm{m}$ emission by using the observed [O IV]25.89 $\mu \mathrm{m} /[\mathrm{Ne}$ III] $15.56 \mu \mathrm{m}$ ratio and subtracting the estimated AGN contribution from the total [Ne III] $15.56 \mu \mathrm{m}$ line strength. Further details are provided in the Appendix. We used the [O IV $] 25.89 \mu \mathrm{m} /[\mathrm{Ne}$ III] $15.56 \mu \mathrm{m}$ ratio instead of the $[\mathrm{O}$ IV $] 25.89 \mu \mathrm{m} /[\mathrm{Ne}$ II] $12.81 \mu \mathrm{m}$ ratio because we could not calculate the typical AGN [O IV] $25.89 \mu \mathrm{m} /$ $[\mathrm{Ne}$ II $] 12.81 \mu \mathrm{m}$ ratio due to the larger relative contribution of SF activity to the [Ne II]12.81 $\mu \mathrm{m}$ emission (Section 4.2). We only subtract this contribution from those active galaxies more than $1 \sigma$ above the typical AGN $\left[\mathrm{Ne}_{\mathrm{III}}\right] 15.56 \mu \mathrm{m} /\left[\mathrm{O}_{\mathrm{IV}}\right] 25.89 \mu \mathrm{m}$ ratio (see Section 5.2 and Figure 8), since the galaxies below this ratio are presumably dominated by the AGN and their SF activity, if any, is masked. This criterion basically selects only those active galaxies with at least $25 \%$ of the [Ne III] $15.56 \mu \mathrm{m}$ emission arising from SF. In our sample $45 \%$ of the AGNs with the [O IV] $25.89 \mu \mathrm{m}$ line detected have excess [Ne III] $15.56 \mu \mathrm{m}$ emission due to SF. The right panel of Figure 10 shows that the fraction of Seyfert 2's with SF is slightly higher than that of Seyfert 1's. In all the luminosity bins except for one, the difference in the fraction with a [Ne III]15.56 $\mu \mathrm{m}$ excess is less than $2 \sigma$ significant. However, for the overall proportion of [Ne III $] 15.56 \mu \mathrm{m}$ excess detections, Fisher's exact test indicates that there is a probability of less than 0.03 that the incidence of this behavior is the same in the Sy1 and Sy 2 samples. Therefore, the full sample in our study supports, with a moderate statistical significance, previous indications that Sy2 host galaxies tend to have higher rates of SF than Sy1 hosts. However, the full sample is heterogeneous and may be subject to a number of biases. For example, it includes a number of radio galaxies, which are known to have low SFRs - in fact, none of the radio galaxies have [Ne III] $15.56 \mu \mathrm{m}$ excess. It also contains ULIRGs with Seyfert spectral characteristics, most of which do have [Ne III]15.56 $\mu \mathrm{m}$ excess. The observed differences depend to some extent on the relative number of galaxies in these two classes. In the following section, we discuss this trend in two relatively complete but smaller subsamples where the biases should be reduced.

\subsection{Results for the $12 \mu \mathrm{m}$ Sample and the RSA Samples}

At this point, we study separately the $12 \mu \mathrm{m}$ sample and the RSA sample. Both are complete samples of Seyfert galaxies selected with homogeneous criteria. The former is selected based on their IRAS $12 \mu \mathrm{m}$ fluxes whereas the latter is selected based on the optical magnitude of the host galaxy. Given the different selection criteria they might be affected by SF in different ways.

As can be seen from Table 4 and Figure 11, the fraction of galaxies with a [Ne III]15.56 $\mu \mathrm{m}$ excess in the RSA sample is higher (by a factor of 1.4) than in the $12 \mu \mathrm{m}$ sample. The lower number of galaxies with a [Ne III]15.56 $\mu \mathrm{m}$ excess in the $12 \mu \mathrm{m}$ sample is because the galaxies of this sample are brighter and the sensitivity to SF of our method is reduced at high [O IV] $25.89 \mu \mathrm{m}$ luminosities. Both effects are clearly seen in Figure 11. First, the RSA sample contains a larger fraction of 

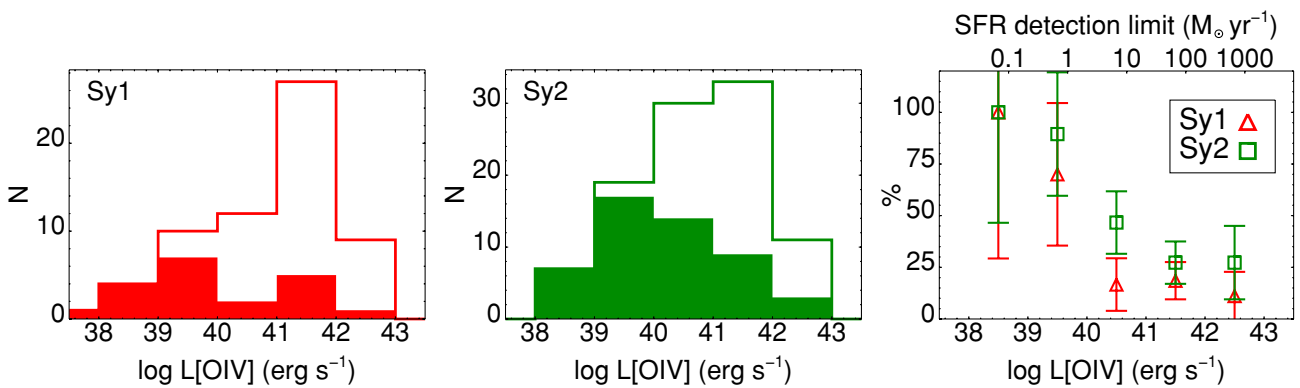

Figure 10. Distribution of the [O IV] $25.89 \mu \mathrm{m}$ luminosities of the Seyfert 1 (left) and 2 (middle) galaxies. The filled histograms are the galaxies with a [Ne III] $15.56 \mu \mathrm{m}$ excess. The right panel compares the fraction of type 1 and 2 Seyferts with a [Ne III]15.56 $\mu \mathrm{m}$ excess for each luminosity bin. We used Poisson statistical errors to estimate the uncertainties of the fractions. The scale on the upper part of the right panel gives the lower limit to the SFR that can be detected with our method for each AGN luminosity bin.

(A color version of this figure is available in the online journal.)

Table 4

Fraction of Galaxies with an [Ne III] Excess in the RSA and $12 \mu \mathrm{m}$ Samples

\begin{tabular}{lcc}
\hline \hline Type & $12 \mu \mathrm{m} \mathrm{Sample}$ & RSA \\
\hline Seyfert 1 & $15 / 45$ & $12 / 25$ \\
Seyfert 2 & $23 / 52$ & $25 / 45$ \\
Total & $38 / 97$ & $37 / 70$ \\
\hline
\end{tabular}

Notes. In this table, we only include galaxies with the [O IV]25.89 $\mu \mathrm{m}$ and [Ne III]15.56 $\mu \mathrm{m}$ emission lines detected. Some galaxies are common to both samples.

low-luminosity Seyferts $\left(L_{[\mathrm{O} \text { Iv }]} \leqslant 10^{40} \mathrm{erg} \mathrm{s}^{-1}\right.$ ) than the $12 \mu \mathrm{m}$ sample. Second, for each AGN luminosity, we are sensitive to a different SFR limit. In other words, for the brightest Seyferts we are only sensitive to SFR above $100 M_{\odot} \mathrm{yr}^{-1}$, while for low-luminosity AGNs we are sensitive to SFR $>0.1 M_{\odot} \mathrm{yr}^{-1}$. We note that the fraction of galaxies with SF in each luminosity bin is similar for both samples (right panel of Figure 11).

In both samples the fraction of Seyfert 2's with [Ne III] $15.56 \mu \mathrm{m}$ excess is slightly larger than that of Seyfert 1's. However, due to the small size of the samples the statistical significance of this excess is low. Based on the two-sample Kolmogorov-Smirnov test, the probability for these differences being due to chance is 0.25 and 0.4 for the $12 \mu \mathrm{m}$ and RSA samples, respectively.

\section{LINE RATIOS AND MODELS}

We studied the physical conditions in the NLR of the AGNs with the photoionization code MAPPINGSIII (Groves et al. 2004). We used a radiation pressure dominated model that includes the effects of dust. For the input parameters we followed the prescription given by Groves et al. (2006). Briefly, we assumed a plane parallel geometry and solar abundances. We modeled the input ionizing spectrum with two power laws with exponential cutoffs (Nagao et al. 2001). We explored the effect of the variations in the total hydrogen column density ranging from $\log n_{\mathrm{H}}\left(\mathrm{cm}^{-2}\right)=19.0$ to 22.0. This range was chosen since it reproduces the observed line ratios and is in good agreement with the hydrogen column density determined using UV and X-ray observations (Crenshaw et al. 2003). For each hydrogen column density, we varied the total pressure $(P / k)$ and the incident ionizing flux $\left(I_{0}\right)$. The values for the total pressure are log $P / k\left(\mathrm{~K} \mathrm{~cm}^{-3}\right)=6,7,8,9$. These correspond to electron densities, as traced by $\left[\mathrm{S}_{\mathrm{II}}\right] \lambda 6716 \AA / \lambda 6731 \AA$, of $\sim<10^{1}, 10^{2}, 10^{3}$, and $>10^{4} \mathrm{~cm}^{-3}$, respectively. The explored range for the ionizing flux is $I_{0}=0.1$ to 0.55 . The incident ionizing flux is scaled
Table 5

Extinction at $24 \mu \mathrm{m}$ Relative to that at $14 \mu \mathrm{m}$

\begin{tabular}{|c|c|}
\hline Extinction Law & $A_{24} / A_{14}$ \\
\hline Rosenthal $^{\mathrm{a}}$ & 1.25 \\
\hline McClure $0.3<A_{\mathrm{K}}<1^{\mathrm{b}}$ & 0.83 \\
\hline McClure $1<A_{\mathrm{K}}<7^{\mathrm{b}}$ & 0.83 \\
\hline Chiar Galactic Center ${ }^{c}$ & 1.38 \\
\hline Chiar Local ISM $^{\mathrm{c}}$ & 0.97 \\
\hline
\end{tabular}

Notes.

${ }^{a}$ Rosenthal et al. (2000).

${ }^{\mathrm{b}}$ McClure (2009).

${ }^{\mathrm{c}}$ Chiar \& Tielens (2006).

by the factor $2.416 \times\left((P / k) / 10^{6}\right) \mathrm{erg}^{-2} \mathrm{sm}^{-1}$ which gives a range in the ionization parameter, $\log U$, from $\sim-3$ to -2 .

\subsection{The [Ne v] Ratio}

Figure 12 shows the ratio between the two [Ne v] emission lines available in the mid-IR. The critical densities of the $[\mathrm{Ne} \mathrm{v}] 14.32 \mu \mathrm{m}$ and $[\mathrm{Ne} \mathrm{v}] 24.32 \mu \mathrm{m}$ lines are $3.5 \times 10^{4} \mathrm{~cm}^{-3}$ and $6.2 \times 10^{3} \mathrm{~cm}^{-3}$, respectively (Osterbrock \& Ferland 2006); thus, they can be used to trace the density of the NLR. We find, however, that a large fraction of the galaxies $(\sim 30 \%)$ lie above the low-density limit ratio. This issue and its possible cause are discussed in detail by Dudik et al. (2007) and also addressed by Tommasin et al. (2010) for a sample of Seyfert galaxies. Dudik et al. (2007) concluded that the ratios above the low-density limit are due to differential extinction from the obscuring torus, and thus can indicate the inclination angle to our line of sight. However, similar to Baum et al. (2010), we do not find any significant difference between the proportion of Seyfert 1 and 2 galaxies above the low-density limit, as would be predicted by this hypothesis.

We calculated the $A_{24.3} / A_{14.3}$ ratio to quantify the extinction needed to move the points above the low-density limit ratio to this value (which would be a lower limit to the extinction since the real ratio may be lower than the low-density limit). The ratios are summarized in Table 5 for some IR extinction laws. As can be seen depending on the extinction law chosen, the extinction can increase or decrease the $[\mathrm{Ne} \mathrm{v}] 24.32 \mu \mathrm{m} /[\mathrm{Ne} \mathrm{v}] 14.32 \mu \mathrm{m}$ ratio. Since the construction of an extinction law implies some interpolation we also used direct measurements of $A_{24} / A_{\mathrm{K}}$ and $A_{15} / A_{\mathrm{K}}$. ${ }^{7}$ Jiang et al. (2006) measured the extinction at

\footnotetext{
7 From the extinction laws, the difference between these $A$ values and those at $14.3 \mu \mathrm{m}$ and $24.3 \mu \mathrm{m}$ would be small $(\sim 5 \%)$.
} 

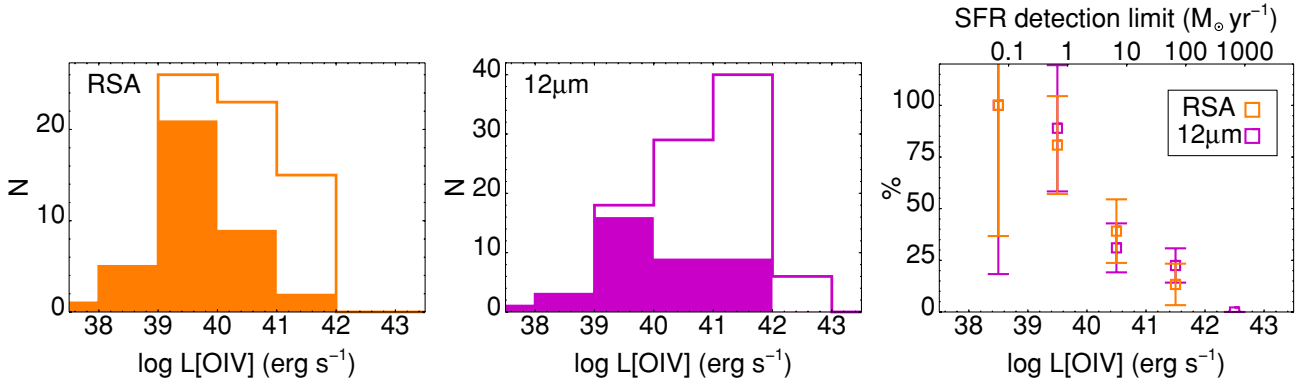

Figure 11. Distribution of the [O Iv] $25.89 \mu \mathrm{m}$ luminosities for the RSA sample (left panel) and $12 \mu \mathrm{m}$ sample (middle panel). Symbols are as in Figure 10. (A color version of this figure is available in the online journal.)

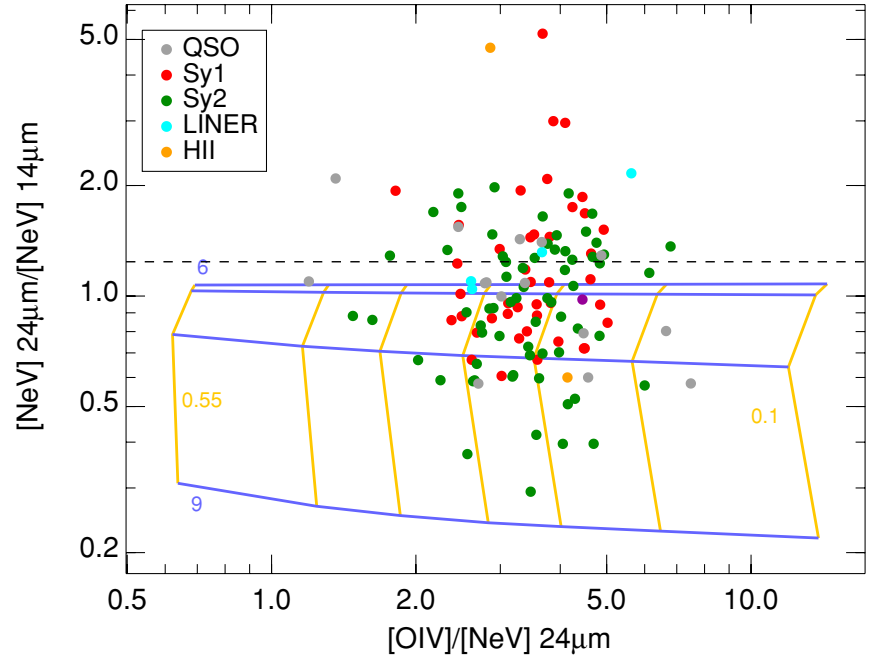

Figure 12. Predicted $[\mathrm{Ne} v[24.32 \mu \mathrm{m} /[\mathrm{Ne} \mathrm{v}] 14.32 \mu \mathrm{m}$ ratio vs. $[\mathrm{O}$ IV $] 25.89 \mu \mathrm{m} /[\mathrm{Ne} \mathrm{v}] 24.32 \mu \mathrm{m}$. Galaxy symbols are as in Figure 2. The dashed black line indicates the low-density limit calculated at $10,000 \mathrm{~K}$ for the $[\mathrm{Ne} \mathrm{v}] 24.32 \mu \mathrm{m} /[\mathrm{Ne} \mathrm{v}] 14.32 \mu \mathrm{m}$ ratio (galaxies above this line have densities below the low-density limit). The blue lines trace constant pressure and the orange lines trace constant ionization parameter $\left(I_{0}\right)$. The input parameters for the model are $\log P / k=6,7,8,9$ and $I_{0}=0.1,0.15,0.20,0.25,0.32,0.4$, 0.55 for constant hydrogen column density $\log n_{\mathrm{H}}\left(\mathrm{cm}^{-2}\right)=20.3$. The galaxy symbols are as in Figure 2.

(A color version of this figure is available in the online journal.)

$15 \mu \mathrm{m}$ and found $A_{15} / A_{\mathrm{K}}=0.40$, although it ranges from 0.25 to 0.55 . For the $A_{24} / A_{\mathrm{K}}$ ratio, the range is 0.28 to 0.65 for different $A_{\mathrm{K}}$ bins (Chapman et al. 2009) and the average value is $\sim 0.5$ (Chapman et al. 2009; Flaherty et al. 2007). From these measurements, we estimate $A_{24.3} / A_{14.3}=1.2 \pm 0.3$. Again, it is close to unity and it is not clear if the extinction would increase or decrease the $[\mathrm{Ne} \mathrm{v}]$ ratio. Moreover, the extinction is nearly neutral between the lines with all the extinction laws and therefore there is very little potential effect on the ratio unless the extinction is extremely large.

We also checked if aperture effects can explain the large values of this ratio. The $[\mathrm{Nev}] 14.32 \mu \mathrm{m}$ line is observed with the Short-High $(\mathrm{SH})$ module $\left(5^{\prime \prime} \cdot 7 \times 11^{\prime \prime} .3\right)$, whereas the $[\mathrm{Ne} \mathrm{v}] 24.32 \mu \mathrm{m}$ is observed with the Long-High (LH) module $\left(11^{\prime \prime} .1 \times 22^{\prime \prime} \cdot 3\right)$. We would expect a correlation between the $[\mathrm{Nev}] 24.32 \mu \mathrm{m} /[\mathrm{Nev}] 14.32 \mu \mathrm{m}$ ratio and the distance if the $[\mathrm{Ne}$ v] emission region was larger than the SH slit. However, we do not find this correlation and thus rule out any aperture bias.

Alternatively, the atomic parameters for the $\mathrm{Ne}^{+4}$ ion may not be sufficiently accurate for comparison with these observations, and the calculated low-density limit may be incorrect.

\subsection{Ionization Parameter}

In Figure 12, we plot the model grid together with the data. In this plot we vary the ionizing flux, $I_{0}$, and the total pressure, $P / k$, for a constant total hydrogen column density $\left(\log n_{\mathrm{H}}\left(\mathrm{cm}^{-2}\right)=\right.$ 20.3). We see that the $[\mathrm{Ne} \mathrm{v}]$ ratio can be used to measure the pressure. However, the pressure (or density) values derived from this ratio have some issues, since a considerable fraction of galaxies are above the low-density limit ratio (Section 7.1).

Also, Figure 12 shows that the [O Iv]25.89 $\mu \mathrm{m} /[\mathrm{Ne} \mathrm{v}]$ $24.32 \mu \mathrm{m}$ ratio traces the ionization parameter. The [O Iv $] 25.89 \mu \mathrm{m} /[\mathrm{Ne} \mathrm{v}] 24.32 \mu \mathrm{m}$ ratio spans a narrow range (less than 1 dex) which is related to the tight correlation found between the [O IV] $25.89 \mu \mathrm{m}$ and [Ne v]24.32 $\mu \mathrm{m}$ luminosities (Section 4). This implies a very small range for the ionization parameter $(-2.8<\log U<-2.5)$. This range is similar to that found when modeling the optical [O III] and $\mathrm{H} \beta$ emission (Baskin \& Laor 2005; Kraemer et al. 1999). The critical density of the [O III] lines is larger than that of these mid-IR lines and for instance, the Baskin \& Laor (2005) models predict electron densities $\log n_{\mathrm{e}}\left(\mathrm{cm}^{-3}\right)=5.8 \pm 0.7$, well above the critical density of the mid-IR lines studied here. Thus, it is possible that these lines are produced in different gas clouds.

\subsection{Hydrogen Column Density}

We use the [Ne III] $15.56 \mu \mathrm{m}$ and the [Ne v]14.32 $\mu \mathrm{m}$ lines to estimate the total hydrogen ( $\mathrm{H}+\mathrm{H}$ II) column density, $n_{\mathrm{H}}$. The [Ne v]14.32 $\mu \mathrm{m}$ line is produced in the inner part of the NLR, while the $[\mathrm{Ne}$ III] $15.56 \mu \mathrm{m}$ line is produced in a more external region. Thus, the $[\mathrm{Ne} I I I] 15.56 \mu \mathrm{m} /[\mathrm{Ne} \mathrm{v}] 14.32 \mu \mathrm{m}$ ratio is subject to change depending on the column density. As expected, the models predict larger $[\mathrm{Ne}$ III $] 15.56 \mu \mathrm{m} /[\mathrm{Ne} \mathrm{v}] 14.32 \mu \mathrm{m}$ ratios for larger $n_{\mathrm{H}}$.

In Figure 13, we plot the model grid together with the observed ratios. The [Ne III] $15.56 \mu \mathrm{m} /[\mathrm{Ne} \mathrm{v}] 14.32 \mu \mathrm{m}$ ratio is not sensitive to column densities larger than $\log n_{\mathrm{H}}\left(\mathrm{cm}^{-2}\right)>21$. We find that the column density for most of the galaxies is in the range $20.3<\log n_{\mathrm{H}}\left(\mathrm{cm}^{-2}\right)<21$. There is no difference between the predicted hydrogen column density for type 1 and 2 galaxies. This column density range is compatible with the values derived from UV observations although lower than that determined using X-ray data (Crenshaw et al. 2003). A few galaxies are above the model grid. The [Ne III] $15.56 \mu \mathrm{m} /[\mathrm{Ne} \mathrm{v}] 14.32 \mu \mathrm{m}$ ratio of these galaxies is larger than that predicted by the models. As we proposed in Sections 4.2 and 5.2, SF acting in these galaxies is likely producing the extra [Ne III] $15.56 \mu \mathrm{m}$ emission. 


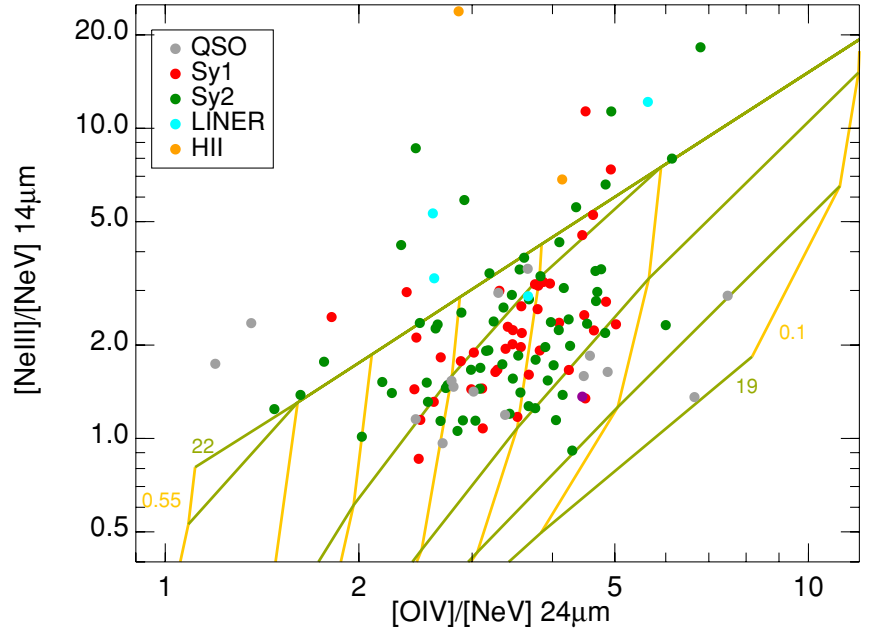

Figure 13. Predicted $[\mathrm{Ne} \mathrm{III}] 15.56 \mu \mathrm{m} /[\mathrm{Ne} \mathrm{v}] 14.32 \mu \mathrm{m}$ ratio vs. $[\mathrm{O}$ IV $] 25.89 \mu \mathrm{m} /[\mathrm{Ne} \mathrm{v}] 24.32 \mu \mathrm{m}$. The green lines trace constant hydrogen column density and the orange lines trace constant ionization parameter. The input parameters for the model are $\log n_{\mathrm{H}}\left(\mathrm{cm}^{-2}\right)=19.0,20.0,20.3,20.6,21.0,22.0$ and $I_{0}=0.1,0.15,0.20,0.25,0.32,0.4,0.55$ for constant pressure $(\log P / k=$ 8). The galaxy symbols are as in Figure 2.

(A color version of this figure is available in the online journal.)

\section{CONCLUSIONS}

We studied a sample of 426 galaxies observed with the Spitzer/IRS spectrograph in the high-resolution mode. Our analysis includes published data for QSO, Seyfert, LINER, and $\mathrm{H}_{\mathrm{II}}$ galaxies as well as unpublished measurements for the RSA Seyfert sample and LIRGs. We explored the relationship between the high-ionization ([Ne v]14.32 $\mu \mathrm{m}$, $[\mathrm{NeV}] 24.32 \mu \mathrm{m}$, and [O IV] $25.89 \mu \mathrm{m})$ and intermediateionization ([Ne III]15.56 $\mu \mathrm{m}$ and $[\mathrm{Ne}$ II] $12.81 \mu \mathrm{m}$ ) emission lines present in the mid-IR spectra. The median ratios for each class of galaxies are listed in Table 3 . The main results are as follows.

1. There is a tight linear correlation between the fluxes and luminosities of the high-ionization emission lines $\left[\mathrm{O}_{\mathrm{IV}}\right] 25.89 \mu \mathrm{m}$ and $[\mathrm{Ne} \mathrm{v}] 24.32 \mu \mathrm{m}$. This correlation spans five orders of magnitude $\left(38<\log L_{[\mathrm{Nev}] 24}\right.$ $\left(\right.$ erg $\left.\mathrm{s}^{-1}\right)<43$ ), ranging from LINERs, low-luminosity Seyfert galaxies, types 1 and 2, to QSOs. The typical $[\mathrm{O}$ IV $] 25.89 \mu \mathrm{m} /[\mathrm{Ne} \mathrm{v}] 24.32 \mu \mathrm{m}$ ratio for AGNs is 3.5, with rms scatter of 0.8 . The correlation also holds between the [O IV] $25.89 \mu \mathrm{m}$ and the [Ne v]14.32 $\mu \mathrm{m}$ lines, although the scatter is larger. The typical [O IV] $25.89 \mu \mathrm{m} /$ $[\mathrm{Ne}$ V]14.32 $\mu \mathrm{m}$ ratio for AGNs is 3.4 , with rms scatter of 1.4 .

2. There is also a good linear correlation between the $[\mathrm{Ne}$ III] $15.56 \mu \mathrm{m}$ and the $[\mathrm{Ne} \mathrm{v}] 14.32 \mu \mathrm{m}$ fluxes and luminosities for Seyfert galaxies and QSOs. The $[\mathrm{Ne}$ III $] 15.56 \mu \mathrm{m} /[\mathrm{Ne} \mathrm{v}] 14.32 \mu \mathrm{m}$ ratio is 1.9 , with $\mathrm{rms}$ scatter of 0.8 . Using this correlation and the previous one, we calculated the typical [Ne III] $15.56 \mu \mathrm{m} /[\mathrm{O}$ IV $] 25.89 \mu \mathrm{m}$ ratio for AGNs of 0.6 , with rms scatter of 0.3 .

3. We calculate the [O IV $] 25.89 \mu \mathrm{m}$ emission due to $\mathrm{SF}$ $\left(\log L_{\text {[O Iv] }}\left(\mathrm{erg} \mathrm{s}^{-1}\right)<\log L_{\mathrm{IR}}\left(\mathrm{erg} \mathrm{s}^{-1}\right)-4.7 \pm 0.6\right)$ and estimate that it may dominate the total [O IV] $25.89 \mu \mathrm{m}$ emission when the intrinsic AGN luminosity is a factor of 20 smaller than the star-forming luminosity. In general, we find that the [O IV]25.89 $\mu \mathrm{m}$ emission is dominated by the AGN in Seyfert galaxies, whereas SF can explain the [O IV]25.89 $\mu \mathrm{m}$ emission of optically classified H II galaxies.

4. We do not find any significant difference between the midIR high-ionization emission lines in type 1 and 2 Seyfert galaxies. Either there are no differences in the conditions in the NLR of the two AGN types, or the effects associated with the different line of sight (i.e., dust extinction) are minimized in the mid-IR spectra.

5. We find that a significant number of Seyfert galaxies from the $12 \mu \mathrm{m}$ sample $(30 \%)$ and the RSA sample (40\%) show evidence for excess [Ne III] $15.56 \mu \mathrm{m}$ emission relative to their [O IV] $25.89 \mu \mathrm{m}$ emission associated with SF. The larger fraction in the RSA sample is explained because the RSA sample contains a larger fraction of low-luminosity AGNs $\left(L_{[\mathrm{O} \text { Iv }]}<10^{41} \mathrm{erg} \mathrm{s}^{-1}\right)$ in which these SF excesses are easier to detect.

6. The fraction of Seyfert 2 galaxies with [Ne III]15.56 $\mu \mathrm{m}$ excess is larger than that of Seyfert 1 with a moderate statistical significance in the full sample although this sample may be affected by several biases. However, when we consider the $12 \mu \mathrm{m}$ or RSA Seyfert samples separately, these differences are not statistically significant due to the smaller size of these samples.

7. A considerable fraction $(30 \%)$ of the galaxies have $[\mathrm{Ne} \mathrm{v}] 24.32 \mu \mathrm{m} /[\mathrm{Ne} \mathrm{v}] 14.32 \mu \mathrm{m}$ ratios above the lowdensity limit. We did not find a connection between the Seyfert type and the ratio of galaxies above the limit. We are not able to explain this in terms of differential extinction.

8. Our modeling shows that the nebular conditions in the NLRs are remarkably similar among all the AGNs in our sample. This similarity allows us to compare conditions critically in the NLRs of type 1 and 2 galaxies. There appear to be no significant overall differences, consistent with the unified model. We constrained the ionization parameter in the range $-2.8<\log U<-2.5$ and the hydrogen column density $20<\log n_{\mathrm{H}}\left(\mathrm{cm}^{-2}\right)<21$.

The relationships presented in this paper provide an important benchmark for the interpretation of the future mid-IR observations of AGNs and star-forming galaxies with JWST/MIRI.

The authors thank B. Groves and T. Díaz-Santos for their help and enlightening discussion. We thank the anonymous referee for useful comments and suggestions. M.P.-S. acknowledges support from the CSIC under grant JAE-Predoc-2007. M.P.-S. also thanks the Steward Observatory for their hospitality during his stay while part of this work was done. A.A.-H. and M.P.-S. acknowledge support from the Spanish Plan Nacional del Espacio under grant ESP2007-65475-C02-01. This work was partially supported by Caltech/JPL through contract 1255094 to the University of Arizona. A.A.-H. also acknowledges support for this work from the Spanish Ministry of Science and Innovation through Proyecto Intramural Especial under grant number 200850I003 and from Plan Nacional de Astronomia y Astrofisica under grant number AYA2009-05705-E. This research has made use of the NASA/ IPAC Extragalactic Database (NED) which is operated by the Jet Propulsion Laboratory, California Institute of Technology, under contract with the National Aeronautics and Space Administration. This research also used the VizieR catalog Service (Ochsenbein et al. 2000) 


\section{APPENDIX}

\section{CALCULATING THE AGN CONTRIBUTION}

In this appendix, we explain briefly the method used in Sections 5 and 6 to calculate the SF fraction of the [Ne III] $15.56 \mu \mathrm{m}$ emission.

The total [Ne III]15.56 $\mu \mathrm{m}$ emission includes two components, one from the AGN and the other from SF:

$$
[\mathrm{Ne} I I I]=[\mathrm{Ne} I I I]_{\mathrm{SF}}+[\mathrm{Ne} \mathrm{III}]_{\mathrm{AGN}} .
$$

If we assume that all the [O IV]25.89 $\mu \mathrm{m}$ emission is produced by the AGN, we can use the typical [Ne III] $15.56 \mu \mathrm{m} /$ [O IV] $25.89 \mu \mathrm{m}$ ratio observed in Seyfert galaxies with low SF (Figure 8 ) to estimate the amount of [Ne III]15.56 $\mu \mathrm{m}$ emission coming from the AGN:

$$
[\mathrm{Ne} \text { III }]=\left[\mathrm{Ne}_{\mathrm{III}}\right]_{\mathrm{SF}}+\left[\mathrm{O}_{\mathrm{IV}}\right] \times\left(\frac{\left[\mathrm{Ne}_{\mathrm{III}}\right]}{\left[\mathrm{O}_{\mathrm{IV}}\right]}\right)_{\mathrm{AGN}} .
$$

Finally, we obtain the fraction of [Ne III]15.56 $\mu \mathrm{m}$ emission from SF:

$$
\frac{\left[\mathrm{Ne}_{\mathrm{III}}\right]_{\mathrm{SF}}}{\left[\mathrm{Ne}_{\mathrm{III}}\right]}=1-\frac{\left[\mathrm{O}_{\mathrm{IV}}\right]}{\left[\mathrm{Ne}_{\mathrm{III}}\right]} \times\left(\frac{[\mathrm{Ne} \mathrm{III}]}{\left[\mathrm{O}_{\mathrm{IV}}\right]}\right)_{\mathrm{AGN}} .
$$

This method can be applied as well to other line ratios. The only assumption is that one of the lines in the ratio is uniquely produced by the AGN while the SF and AGN contribute to the other line (e.g., the [Ne III] $15.56 \mu \mathrm{m}$ and [Ne v] lines). Also note that this method makes use of a typical AGN ratio; thus, the estimated SF contribution to an emission line for a single object will be uncertain and dependent on the ionization parameter.

\section{REFERENCES}

Allen, M. G., Groves, B. A., Dopita, M. A., Sutherland, R. S., \& Kewley, L. J. 2008, ApJS, 178, 20

Alonso-Herrero, A., Quillen, A. C., Rieke, G. H., Ivanov, V. D., \& Efstathiou, A. 2003, AJ, 126, 81

Alonso-Herrero, A., Rieke, G. H., Rieke, M. J., Colina, L., Pérez-González, P. G., \& Ryder, S. D. 2006, ApJ, 650, 835

Alonso-Herrero, A., et al. 2009, ApJ, 697, 660

Antonucci, R. 1993, ARA\&A, 31, 473

Armus, L., et al. 2007, ApJ, 656, 148

Baskin, A., \& Laor, A. 2005, MNRAS, 358, 1043

Baum, S. A., et al. 2010, ApJ, 710, 289

Beirão, P., Brandl, B. R., Devost, D., Smith, J. D., Hao, L., \& Houck, J. R. 2006, ApJ, 643, L1

Beirão, P., et al. 2008, ApJ, 676, 304

Bernard-Salas, J., et al. 2009, ApJS, 184, 230

Brandl, B. R., et al. 2006, ApJ, 653, 1129

Buchanan, C. L., Gallimore, J. F., O’Dea, C. P., Baum, S. A., Axon, D. J., Robinson, A., Elitzur, M., \& Elvis, M. 2006, AJ, 132, 401

Chapman, N. L., Mundy, L. G., Lai, S., \& Evans, N. J. 2009, ApJ, 690, 496

Chiar, J. E., \& Tielens, A. G. G. M. 2006, ApJ, 637, 774

Crenshaw, D. M., Kraemer, S. B., \& George, I. M. 2003, ARA\&A, 41, 117

Crowther, P. A., Beck, S. C., Willis, A. J., Conti, P. S., Morris, P. W., \& Sutherland, R. S. 1999, MNRAS, 304, 654

Dale, D. A., et al. 2009, ApJ, 693, 1821

Deo, R. P., Crenshaw, D. M., Kraemer, S. B., Dietrich, M., Elitzur, M., Teplitz, H., \& Turner, T. J. 2007, ApJ, 671, 124

Diamond-Stanic, A. M., \& Rieke, G. H. 2010, ApJ, 724, 140

Diamond-Stanic, A. M., Rieke, G. H., \& Rigby, J. R. 2009, ApJ, 698, 623

Díaz-Santos, T., Alonso-Herrero, A., Colina, L., Packham, C., Levenson, N. A., Pereira-Santaella, M., Roche, P. F., \& Telesco, C. M. 2010, ApJ, 711, 328

Dudik, R. P., Satyapal, S., \& Marcu, D. 2009, ApJ, 691, 1501

Dudik, R. P., Weingartner, J. C., Satyapal, S., Fischer, J., Dudley, C. C., \& O’Halloran, B. 2007, ApJ, 664, 71
Farrah, D., et al. 2007, ApJ, 667, 149

Flaherty, K. M., Pipher, J. L., Megeath, S. T., Winston, E. M., Gutermuth, R. A., Muzerolle, J., Allen, L. E., \& Fazio, G. G. 2007, ApJ, 663, 1069

Genzel, R., et al. 1998, ApJ, 498, 579

Gorjian, V., Cleary, K., Werner, M. W., \& Lawrence, C. R. 2007, ApJ, 655, L73 Goulding, A. D., \& Alexander, D. M. 2009, MNRAS, 398, 1165

Groves, B. A., Dopita, M. A., \& Sutherland, R. S. 2004, ApJS, 153, 9

Groves, B., Dopita, M., \& Sutherland, R. 2006, A\&A, 458, 405

Ho, L. C., Filippenko, A. V., \& Sargent, W. L. W. 1997, ApJS, 112, 315

Ho, L. C., \& Keto, E. 2007, ApJ, 658, 314

Houck, J. R., et al. 2004, ApJS, 154, 18

Imanishi, M., \& Wada, K. 2004, ApJ, 617, 214

Jiang, B. W., Gao, J., Omont, A., Schuller, F., \& Simon, G. 2006, A\&A, 446, 551

Kauffmann, G., et al. 2003, MNRAS, 346, 1055

Kraemer, S. B., Turner, T. J., Crenshaw, D. M., \& George, I. M. 1999, ApJ, 519, 69

Lutz, D., Kunze, D., Spoon, H. W. W., \& Thornley, M. D. 1998a, A\&A, 333, L75

Lutz, D., Spoon, H. W. W., Rigopoulou, D., Moorwood, A. F. M., \& Genzel, R. 1998b, ApJ, 505, L103

Maiolino, R., \& Rieke, G. H. 1995, ApJ, 454, 95

Maiolino, R., Ruiz, M., Rieke, G. H., \& Keller, L. D. 1995, ApJ, 446, 561

McClure, M. 2009, ApJ, 693, L81

Meléndez, M., Kraemer, S. B., Schmitt, H. R., Crenshaw, D. M., Deo, R. P., Mushotzky, R. F., \& Bruhweiler, F. C. 2008a, ApJ, 689, 95

Meléndez, M., et al. 2008b, ApJ, 682, 94

Nagao, T., Murayama, T., \& Taniguchi, Y. 2001, ApJ, 546, 744

Ochsenbein, F., Bauer, P., \& Marcout, J. 2000, A\&AS, 143, 23

Ogle, P., Whysong, D., \& Antonucci, R. 2006, ApJ, 647, 161

Oliva, E., Moorwood, A. F. M., Drapatz, S., Lutz, D., \& Sturm, E. 1999, A\&A, 343, 943

Osterbrock, D. E., \& Ferland, G. J. (ed.) 2006, in Astrophysics of Gaseous Nebulae and Active Galactic Nuclei (2nd ed.; Sausalito, CA: Univ. Science Books)

Peeters, E., Spoon, H. W. W., \& Tielens, A. G. G. M. 2004, ApJ, 613, 986

Pereira-Santaella, M., Alonso-Herrero, A., Rieke, G. H., Colina, L., DíazSantos, T., Smith, J.-D. T., Pérez-González, P. G., \& Engelbracht, C. W. 2010, ApJS, 188, 447

Pottasch, S. R., Surendiranath, R., Bernard-Salas, J., \& Roellig, T. L. 2009, A\&A, 502, 189

Ramos Almeida, C., et al. 2009, ApJ, 702, 1127

Rigby, J. R., Diamond-Stanic, A. M., \& Aniano, G. 2009, ApJ, 700, 1878

Rigby, J. R., \& Rieke, G. H. 2004, ApJ, 606, 237

Roche, P. F., Aitken, D. K., Smith, C. H., \& Ward, M. J. 1991, MNRAS, 248, 606

Rosenthal, D., Bertoldi, F., \& Drapatz, S. 2000, A\&A, 356, 705

Rush, B., Malkan, M. A., \& Spinoglio, L. 1993, ApJS, 89, 1

Sanders, D. B., Mazzarella, J. M., Kim, D.-C., Surace, J. A., \& Soifer, B. T. 2003, AJ, 126, 1607

Sanders, D. B., \& Mirabel, I. F. 1996, ARA\&A, 34, 749

Satyapal, S., Sambruna, R. M., \& Dudik, R. P. 2004, A\&A, 414, 825

Satyapal, S., Vega, D., Dudik, R. P., Abel, N. P., \& Heckman, T. 2008, ApJ, 677, 926

Schaerer, D., \& Stasińska, G. 1999, A\&A, 345, L17

Shi, Y., Rieke, G. H., Ogle, P., Jiang, L., \& Diamond-Stanic, A. M. 2009, ApJ, 703, 1107

Smith, J. D. T., Rudnick, L., Delaney, T., Rho, J., Gomez, H., Kozasa, T., Reach, W., \& Isensee, K. 2009, ApJ, 693, 713

Snijders, L., Kewley, L. J., \& van der Werf, P. P. 2007, ApJ, 669, 269

Sturm, E., Lutz, D., Verma, A., Netzer, H., Sternberg, A., Moorwood, A. F. M., Oliva, E., \& Genzel, R. 2002, A\&A, 393, 821

Sturm, E., et al. 2006, ApJ, 653, L13

Surace, J. A., Sanders, D. B., \& Mazzarella, J. M. 2004, AJ, 127, 3235

Takeuchi, T. T., Yoshikawa, K., \& Ishii, T. T. 2003, ApJ, 587, L89

Thornley, M. D., Schreiber, N. M. F., Lutz, D., Genzel, R., Spoon, H. W. W., Kunze, D., \& Sternberg, A. 2000, ApJ, 539, 641

Tommasin, S., Spinoglio, L., Malkan, M. A., \& Fazio, G. 2010, ApJ, 709, 1257

Tommasin, S., Spinoglio, L., Malkan, M. A., Smith, H., González-Alfonso, E., \& Charmandaris, V. 2008, ApJ, 676, 836

Urry, C. M., \& Padovani, P. 1995, PASP, 107, 803

Veilleux, S., et al. 2009, ApJS, 182, 628

Verma, A., Lutz, D., Sturm, E., Sternberg, A., Genzel, R., \& Vacca, W. 2003, A\&A, 403, 829

Weaver, K. A., et al. 2010, ApJ, 716, 1151

Weedman, D. W., et al. 2005, ApJ, 633, 706 